\title{
Quantification of Rare Earth Elements using Laser-Induced Breakdown Spectroscopy
}

${ }^{1}$ Madhavi Martin, ${ }^{2}$ Rodger C. Martin, ${ }^{1}$ Steve Allman, ${ }^{3}$ Deanne Brice,,${ }^{1}$ Ann Wymore, and ${ }^{4}$ Nicolas Andre.

${ }^{1}$ Biological Sciences Division,

${ }^{2}$ Fusion and Materials for Nuclear Systems Division, and

${ }^{3}$ Environmental Sciences Division, Oak Ridge National Laboratory, Oak Ridge, TN 37831

${ }^{4}$ The University of Tennessee, Center for Renewable Carbon, Knoxville, TN 37996

\begin{abstract}
A study of the optical emission as a function of concentration of laser-ablated yttrium (Y) and of six rare earth elements, europium (Eu), gadolinium $(\mathrm{Gd})$, lanthanum (La), praseodymium $(\mathrm{Pr})$, neodymium $(\mathrm{Nd})$, and samarium $(\mathrm{Sm})$, hasbeen evaluated using the laser-induced breakdown spectroscopy (LIBS) technique. Statistical methodology using multivariate analysis has been used to obtain the sampling errors, coefficient of regression, calibration and crossvalidationof measurements as they relate to the LIBS analysis in graphite-matrix pellets that were doped with elements at severalconcentrations. Each element (in oxide form) was mixed in the graphite matrix in percentages ranging from $1 \%$ to $50 \%$ by weight and the LIBS spectra obtained for each composition as well as for pure oxide samples. Finally a single pellet was mixed with all the elements in equal oxide masses to determine if we can identify the elemental peaks in a mixed pellet. This dataset is relevant for future application to studies of fission product content and distribution in irradiated nuclear fuels. These results demonstrate that LIBS
\end{abstract}


technique is inherently well suited for the future challenge of in situ analysis of nuclear materials. These studies also show that LIBS spectral analysis using statistical methodologycan provide quantitative results, and suggest an approach in future to the far more challenging multielementalanalysis of 20 primary elements in high-burnup nuclear reactor fuel.

\section{Introduction}

In nuclear fuel fission reactions, each uranium or plutonium atom will split into two lighter elements. Rare earth elements are a major component of these fission products. Since fission product content in nuclear fuels is proportional to the fraction of "burned" fuel, it can be used to estimate actinide masses (U, Pu, etc.) for nonproliferation "safeguards" monitoring. The reprocessing of irradiated fuel must remove most fission products prior to reuse as fuel.

Therefore, it is important to monitor the presence of mixed actinide, rare earths, and other fission product elements throughout the separation process. Challenges in monitoring fission product content using conventional analytical techniques can be significant, such as in electrochemical recycling processes [1]. The emission spectra of rare earths are very complex and spectral interferences must be known, so a detailed and comprehensive study of the predominant rare earths using LIBS has been undertaken in this research andreported here. There are anumber ofdetailed research articles that have shown the LIBS measurements on actinides, their surrogates,[2-7, 27] and rare earth elements [8-26]. This study is the most comprehensive LIBS analysis of rare earth elements individually and in a mixture.

Prior research shows that the technique of LIBS has been used in the elemental analysis of the rare earth elements [8-27]. Among those, europium has been studied the most. $\mathrm{Eu}^{+}$has 
been detected in colloidal solutions and the technique has been used in the determination of solubility data and of concentrations of europium in glass matrices [8-13]. Another study has determined trace levels of samarium, europium, and gadolinium in aqueous samples by LIBS [9]. Stainless steel coated gadolinium, and gadolinium in its solid oxide form and in molten glass matrices have been studied $[15,16]$. LIBS studies of other rare earths such as lanthanum [14, 17], neodymium [18-22], praseodymium, samarium [27, 23], and yttrium [24-27] have been also been performed.

The rare earths studied here using the LIBS technique are europium (Eu), gadolinium (Gd), lanthanum (La), neodymium (Nd), praseodymium (Pr), samarium ( $\mathrm{Sm})$, and the transition metal yttrium (Y). The rationale for selecting these particular elements for the LIBS studies in this article is given by a typical example: in irradiated nuclear reactor fuel, after decay of 150 days, $\sim 18 \%$ of the fissions result inNd atoms, $13 \%$ in Ce atoms (previously studied using LIBS [28]), 6\% in $\mathrm{La}$ and in $\mathrm{Pr}, 4 \%$ in $\mathrm{Y}$ and in $\mathrm{Sm}, 0.6 \%$ in $\mathrm{Eu}$, and $0.3 \%$ in $\mathrm{Gd}$ [29]. There are numerous difficulties associated with undertaking the simultaneous analysis of actinides, rare earths, and other fission products, and extremely dense and complex spectral features result from the as-produced mixture. These difficulties are (i) experimental, (ii) operational, and (iii) safety related.The technique of choice for measuring the actinide and rare earth content in irradiated fuel is ICP-MS or ICP-OES/AES [30-41], but the challenge of analyzing concentrated solutions from fuel dissolution with very high radiological dose must first be addressed. This creates analytical uncertainties from large dilutions, up to a million-fold, which can be costly and time consuming and increase worker hazards in sample handling. Furthermore, complex spectral features can create problems in identification, fingerprinting, and in the quantification of these elements. Operational difficulties often involveburdensome paperwork, approvals, andcontrols 
that are required when handling even $<1 \mathrm{~g}$ of fissile material such as enriched uranium or plutonium. Setting up glove boxes for radioanalytical work has significant operational costs, and hot cell costs are even higher. Pre-operational safety analysis of new equipment and operations and of radioactive content and its potential for release is far more rigorous than typical laser and chemical safety requirements. In addition to the experimental and operational difficulties, each nuclear facility in the U. S. has "safety basis documents" that set nuclear safety boundaries for experimental activities. Any new hazard introduced into a nuclear facility must be analyzed and documented. This work must be approved prior to beginning new experiments and can take months if not routine.For these reasons, LIBS has certain advantages as a micro-analytical technique in potentially mitigating some of these controls compared to conventional analytical techniques.

As with all analytical techniques, LIBS has inherent limitations that hinder quantification. Due to the matrix effects, true quantitative analyses and calibrations can be a problem.One drawback for the LIBS technique is thatdifferent matrices for different samples require matrixspecific calibration standards. Windom and Hahn have overcome the matrix effects for the LIBS technique in their article [42] and also discussions of the challenges for the quantitative analysis for the LIBS technique have been shown in the review articles by Hahn and Omenetto and other authors [43-45]. Certain issues such as sampling geometry on elemental emissions and other sampling parameters have been clearly addressed [46,47].

For the previously mentioned example of irradiated nuclear reactor fuel after decay of 150 days, the estimates of fission product content [29] indicate that $\sim 23 \%$ of the fission product mass is represented by the seven elements measured in this work, which also provides a spectral database of these elements for further LIBS studies. A previous LIBS study of $\mathrm{Cs}, \mathrm{Sr}$, and $\mathrm{Ce}$ in 
the same matrix as here [28] covers another $\sim 18 \%$ of this fission product mass and associated LIBS spectra. Separate LIBS studies of Mo, Zr (in U-Zr alloy form), and Pd contribute spectral data for another $\sim 24 \%$ of the fission product mass. Spectral data from these separate studies can now be used to plan the LIBS measurement of and interpretation of results for nearly two-thirds of the fission product content (depending on the time of decay prior to measurement) in irradiated fuel samples. Synthesis of these spectral libraries with the spectral tabulations from recent mixed actinide studies [6] of $\mathrm{U}, \mathrm{Np}, \mathrm{Pu}$, and Am now provides a starting point for analysis of complex nuclear fuel mixtures, including selection of optimal low-interference identifying peaks for each element.

In 2003 a feasibility experiment demonstrated LIBS analysis of the dried residue from $25 \mu \mathrm{L}$ of concentrated, undiluted radiochemical process solution containing significant masses of americium, curium, and rare earth and other fission products [48]. No ICP-based instrument could measure this sample without multiple orders-of-magnitude dilution and resultant propagation of analytical uncertainties. Since then, no comparable experiment has been reported in the literature, but the results clearly indicated the need for an a priori comprehensive LIBS spectral library to qualitatively predict spectral interferences and optimize the selection of microvolume and mass content of the pipetted sample to facilitate subsequent data interpretation. Because of the prolific atomic emission lines from the major rare earth fission products (in addition to the even more prolific lines from any actinides present), this work represents a significant step toward experimental planning of futureLIBS analyses of real-world nuclear samples. 
The objective of this article is to:

- obtain the broadband LIBS emission spectra from single-element samples of six rare earth elements $\mathrm{Eu}, \mathrm{Gd}, \mathrm{La}, \mathrm{Nd}, \mathrm{Pr}$, and Sm, and one transition metal Y, at concentrations of $1 \%$ and $50 \%$ by weight for each element in oxide form within a carbon matrix. This has allowed the authors to identify the specific peaks for each element and develop a calibration curve for each.

- develop multivariate calibration and cross-validations for each individual element (shown in table 3) and,

- obtain the broadband LIBS emission spectrum from an equal mixture of the rare earth elements, Eu, Gd, La, Nd, Pr, and Sm, and one transition metal Y to see if individual emission lines from these can be identified in this mixture.

\section{Experimental}

LIBS is a laser-based technique in which, for this study, a laser of $532 \mathrm{~nm}$ wavelength is focused onto the surface of a sample which vaporizes and ionizes a small volume of sample material to generate a plasma spark. All states of matter can be analyzed; e.g., solid, liquid, gaseous, and aerosol. LIBS vaporizes a small sample volume with sufficient energy to ionize all elemental components into optically excited states in the resultant sample plume. The vaporized species then undergo de-excitation and optical emission on a microsecond time scale, and timedependent ultraviolet-visible spectroscopy fingerprints the elements associated with the spectral peaks. The plasma emits light that is collected by an optical fiber and delivered to a spectrometer. The spectrometer separates out the white light of the plasma into different 
wavelengths, and coupling ontothe face of an intensified charge coupled device (ICCD) detector converts the optical signal into an electronic signal. Powerful computer software is used to present a spectrum (intensity versus wavelength) of the sample under test. The process of excitation, light collection, separation into wavelengths, and optical signal conversion to electronic signal is completed within 100 milliseconds. LIBS is typically a surface analytical technique, with each laser pulse vaporizing microgram or submicrogram sample masses. However, the rapidity of sampling (typically $10 \mathrm{~Hz}$ laser repetition rate) and ability to scan a sample surface, ablate a hole into a solid sample with repeated laser pulses (for depth profiling) or focus the laser spark below the surface of a liquid sample permits more versatile analyses and provides sufficient statistics for bulk sampling. The detection limits are often in the parts per million (1 ppm to100 ppm) rangefor mostelements in the periodic table (dependent on sample matrix). These limits of detection can be reduced when the sample is placed underreduced atmospheric pressure or in an inert gaseous environment.

\subsection{Instrumentation}

The main components of the experimental system are a pulsed Big Sky laser model CFRUltra which is a Q-switched Nd:YAG laser with frequency doubled output wavelength of 532 $\mathrm{nm}$ [28]. Laser energy of $45 \mathrm{~mJ}$ was used to obtain all the samplespectra. The experimental setup also has an X-Y-Z stage to provide capability of three-dimensional movements. A displacement laser $(650 \mathrm{~nm})$ is employed to control the vertical position of the sample upper surface, making sure that the laser beam which is exciting the sample surface remains focused and that the plasma is optimally located with respect to the collection optics.The light emitted by the plasma at the focal volume was collected by a set of collection optics and focused into a low $\mathrm{O}-\mathrm{H}$ silica fiber.The optical fiber is used to deliver the light to an Echelle spectrometerfrom 
Catalina Scientific model SE 200 spectrometer that resolveslight into different wavelengths by a high order dispersion module (190-800 nm range), followed by detection using a 2-D 1024x1024 pixel intensified charge coupled detector (ICCD) made by Andor Technologies. The advantage of using an ICCD is that the detection can be delayed with respect to plasma formation. The data was collected by using a delay of 1 microsecond, a gate width of 10 microseconds, and the repetition rate at which the laser was operated was $10 \mathrm{~Hz}$. The whole process of sample excitation, plasma formation, light collection, and optical-to-digital conversion of the signal takes at most a second to a few seconds depending on the number of shots that are averaged to get a good signal-to-noise ratio. Table 1 summarizes the laser and detector/spectrometer characteristics.

Table1. Characteristics of laser and detector/spectrometer.

\begin{tabular}{|l|l|l|l|l|l|}
\hline \multicolumn{7}{|c|}{ Laser Characteristics } \\
\hline \hline Wavelength & Energy/pulse & Rep- rate & & & \\
\hline $532 \mathrm{~nm}$ & $45 \mathrm{~mJ}$ & $10 \mathrm{~Hz}$ & \multicolumn{3}{l|}{} \\
\hline Detector/Spectrometer Characteristics & \multicolumn{3}{|c|}{ Pixel resolution (FWHM) nm } \\
\hline $\begin{array}{l}\text { Wavelength } \\
\text { range nm }\end{array}$ & $\begin{array}{l}\text { Practical } \\
\text { spectral } \\
\text { bandwidth }\end{array}$ & & $200-400$ & $400-600$ & $600-800$ \\
\hline $190-800$ & $210-790 \mathrm{~nm}$ & & 0.06 & $0.06-0.08$ & $0.09-0.12$ \\
\hline
\end{tabular}

\subsection{Reagents}

All the rare earth oxide powders $\left(\mathrm{Eu}_{2} \mathrm{O}_{3}, \mathrm{Gd}_{2} \mathrm{O}_{3}, \mathrm{La}_{2} \mathrm{O}_{3}, \mathrm{Nd}_{2} \mathrm{O}_{3}, \mathrm{Pr}_{6} \mathrm{O}_{11}, \mathrm{Sm}_{2} \mathrm{O}_{3}\right.$, and the transition metal oxide $\mathrm{Y}_{2} \mathrm{O}_{3}$ ), were obtained from Alpha Aesar, Ward Hill, Massachusetts (USA). The purity of the oxide powders that were used in this research was $99.99 \%$. The graphite 
powder that was a natural, microcrystal grade, of $99.9995 \%$ purity was also obtained from Alpha Aesar, Ward Hill, Massachusetts (USA).The polyvinyl acetate (PVA) was 99-100\% hydrolyzed, and was obtained from Acros Organics, New Jersey, USA.

\subsection{Preparation of samples}

The powders of the rare earth oxides $\mathrm{Eu}_{2} \mathrm{O}_{3}, \mathrm{Gd}_{2} \mathrm{O}_{3}, \mathrm{La}_{2} \mathrm{O}_{3}, \mathrm{Nd}_{2} \mathrm{O}_{3}, \mathrm{Pr}_{6} \mathrm{O}_{11}, \mathrm{Sm}_{2} \mathrm{O}_{3}$, and the transition metal oxide $\mathrm{Y}_{2} \mathrm{O}_{3}$ were used in making the pellets. These powders were weighed along witha balancing amount of graphite powderdiluent. The total weight of the oxide material and graphite mixture was $75 \mathrm{mg}$. These componentswerecombined in a bottle and the mixture vortexed and stirred. A quantity of 300 uLof $0.5 \%$ PVA solution was pipetted into a glass tube and re-mixeda second time as in the previous step. A heating block was used to dry the mixed powders containing PVA. The contents of the bottle were emptied into a $1 / 4$ " die and pressed at $15001 \mathrm{~b}$ for at least one minute. The pellets were placed in a labelled plastic bag until subsequent LIBS measurements.

\section{Results and Discussions}

LIBS spectra obtained using an Echelle spectrometer for $1 \%$ by weight of each of the rare earth (Eu, Gd, La, Nd, Pr, and Sm) and Y (transition metal) oxides, mixed with a 99\% balance of graphite powder are shown in figure 1(a). The elemental concentrations range from $0.79 \%$ for $\mathrm{Y}$ to $0.87 \%$ for $\mathrm{Gd}$. The acquired range of the spectra is $200-800 \mathrm{~nm}$ (reduced to $250-700 \mathrm{~nm}$ here), obtained under an argon purge atmosphere. The spectra are very complex with a dense distribution of spectral features that are extremely difficult and tedious to identify individually. Since the buildup of fission product elements from nuclear fission

$1.6 e+5+\quad \begin{aligned} & \text { Carbon and } \\ & \text { C-N Bands }\end{aligned}$




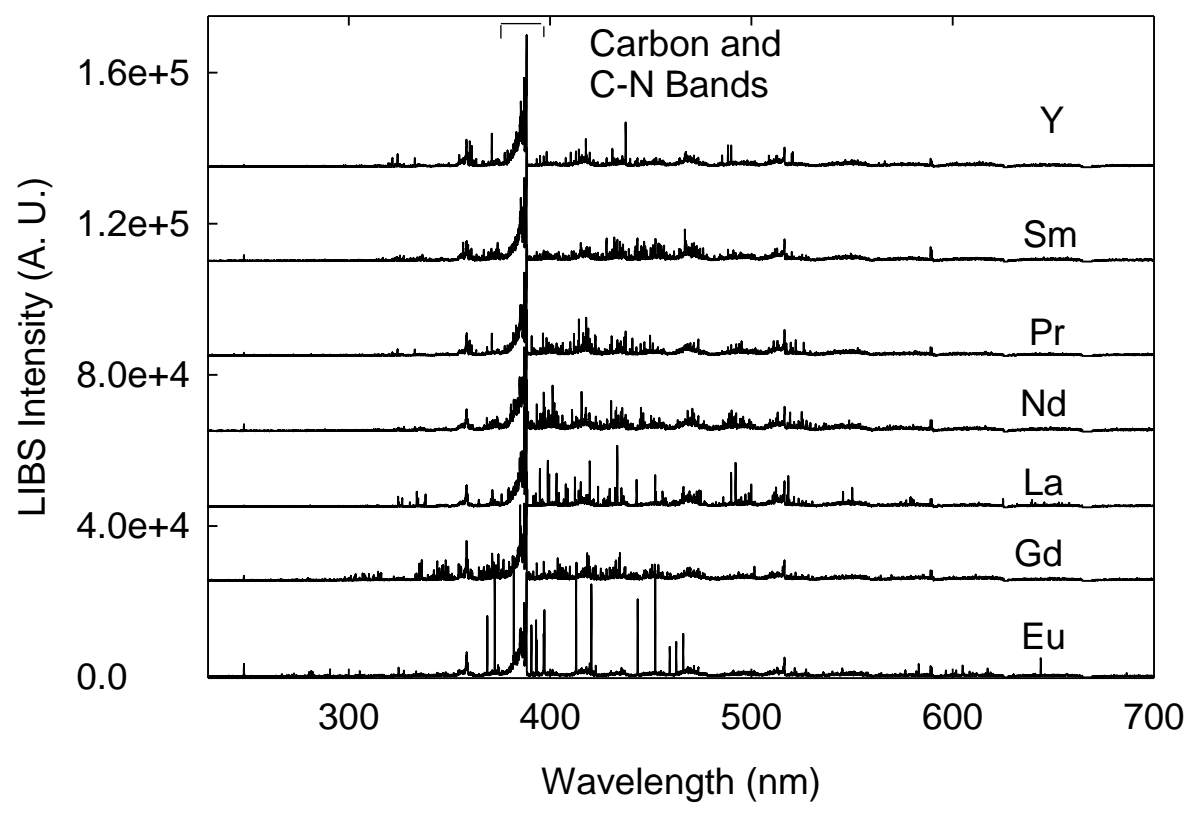

Figure 1 (a).LIBS spectra for $1 \%$ concentration of $\mathrm{Eu}, \mathrm{Gd}, \mathrm{La}, \mathrm{Nd}, \mathrm{Pr}, \mathrm{Sm}$, and Y oxides individually in graphite matrix.

reactions can be as high as $18 \%$ of the total fissions for Nd, figure 1(b) shows the spectra of $25 \%$ by weight of each element's oxide (with elemental concentrations ranging from $19.7 \%$ for $\mathrm{Y}$ to $21.7 \%$ for $\mathrm{Gd}$ ), in which the complexity and density of peaks increase rapidly over the $1 \%$ spectra shown in figure 1(a). By zooming into the spectral region of interest the individual spectral lines can be clearly observedas shown in figure 1(c). Due to the density ofthe numberof 
spectral lines, some line interferences are observed.

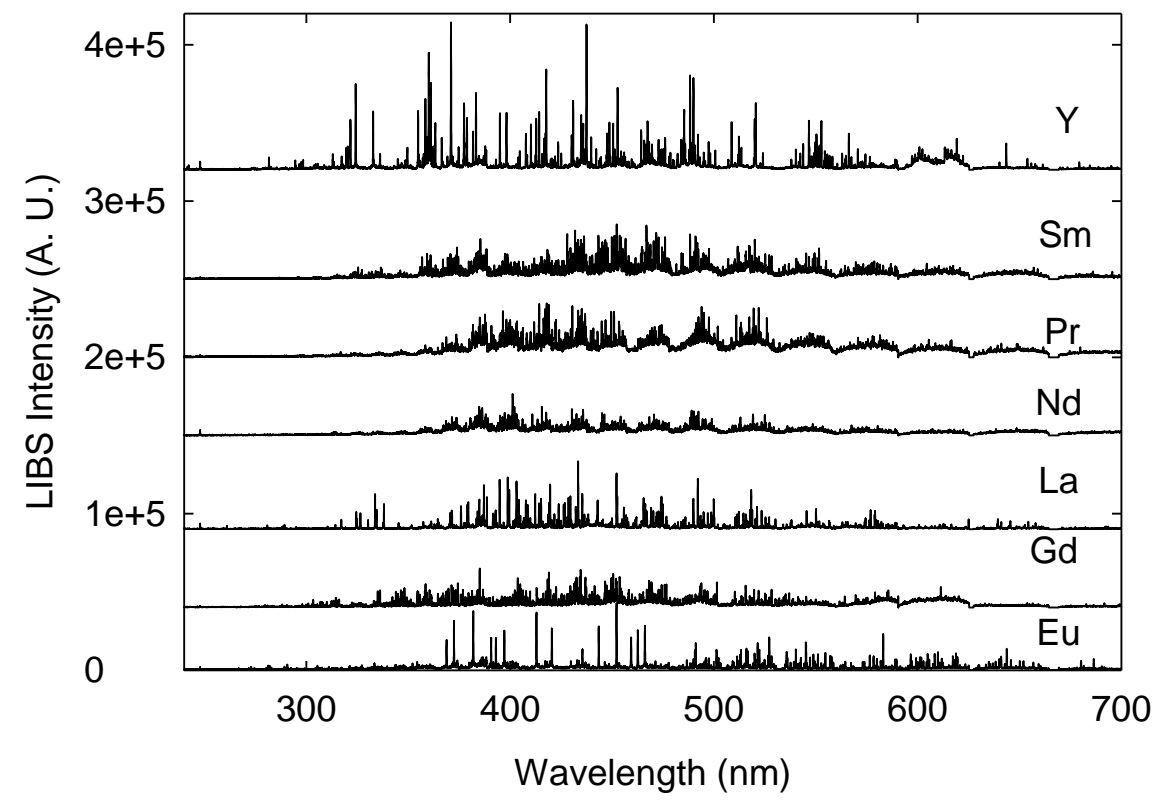

Figure 1 (b).LIBS spectra for $25 \%$ concentration of $\mathrm{Eu}, \mathrm{Gd}, \mathrm{La}, \mathrm{Nd}, \mathrm{Pr}, \mathrm{Sm}$, and Y oxides individually in graphite matrix.

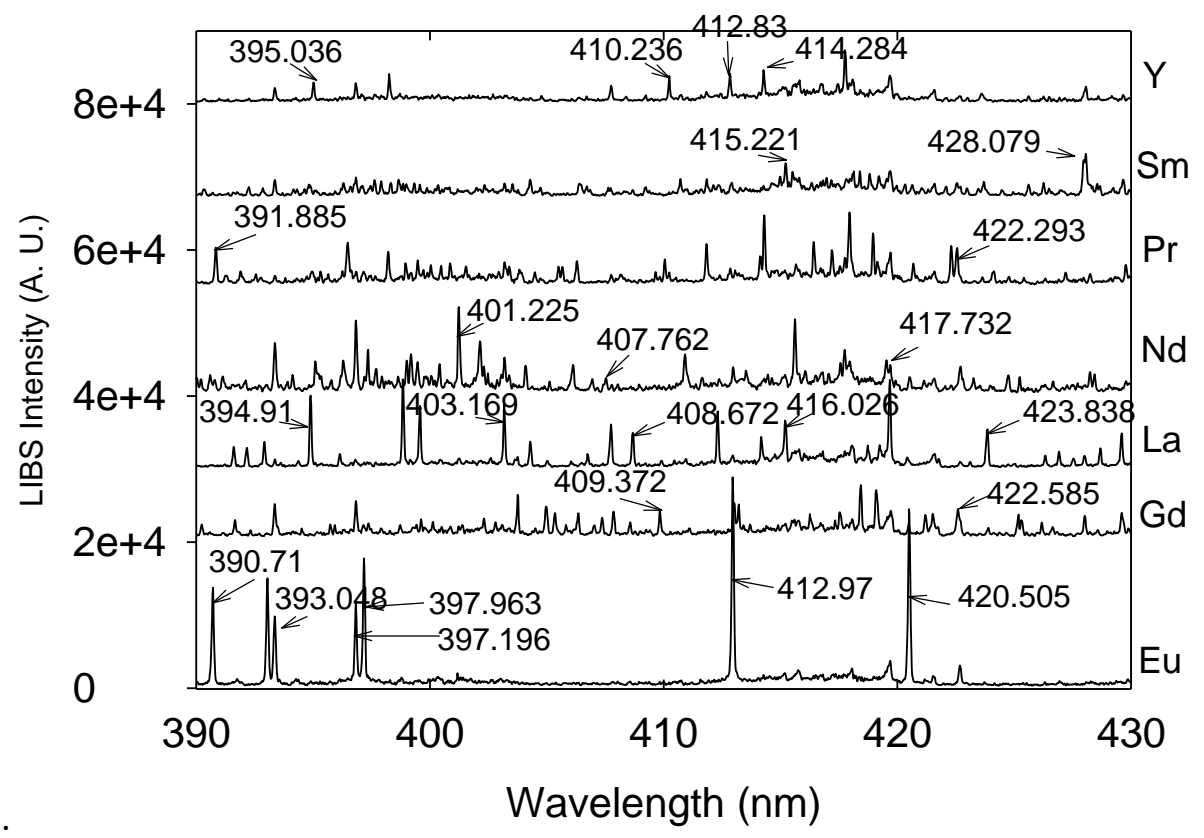


Figure 1 (c).LIBS spectra for $25 \%$ concentration of Eu, Gd, La, Nd, Pr, Sm, and Y oxides in the expanded wavelength window of 390-430 nm.

The main neutral and ionic lines of the rare earths have been identified in the expanded wavelength region $(390-430 \mathrm{~nm})$ in figure 1(c). This spectrum shows that multiple signature peaks for fingerprinting each rare earth element can be usedin calibrating these individual elements. When we correlate the peaks in the LIBS spectra to the NIST-Atomic spectra data base lines, the peaks that were identified for Y are 393.066, 395.036, 398.26, 407.735, 410.236, 412.83, 414.284,417.754, and 419.928nm. The peaks identified for Sm are 392.828,411.019, $411.855,415.221,419.945$, and $428.079 \mathrm{~nm}$. The peaks that were identified for Pr are 391.885 , $396.426,398.051,398.968,399.583,405.88,410.073,411.389,414.311,416.804,417.225$, 417.939, and 422.293 nm. ForNd, the peaks of interests were392.71, 395.745, 397.33, 397.949, $399.01,401.225,402.478,404.08,406.109,407.762,410.946,415.626$, and $417.732 \mathrm{~nm}$. A number of peaks identified for La are 392.922, 394.91, 398.852, 399.575, 403.169, 404.291, $407.735,408.672,412.323,415.197,416.026,419.655$, and $423.838 \mathrm{~nm}$. The peaks that were identified for Gd are 396.929, 404.684, 405.364, 405.822, 407.87, 409.372, 418.425, 419.078, and $422.585 \mathrm{~nm}$ and lastly the peaks correlated to $\mathrm{Eu}$ are $390.71,392.887,393.048,397.196$, 397.963, 412.97, 420.505nm (figure 1(c)). All the peaks that are mentioned here have not been shown in figure 1(c) because of the scaling for the seven spectra it was difficult to label them individually. This shows that even in a small wavelength range, we have a cluster of 70 peaks that can be used to identify definitively the set of seven elements that have been studied for this article 


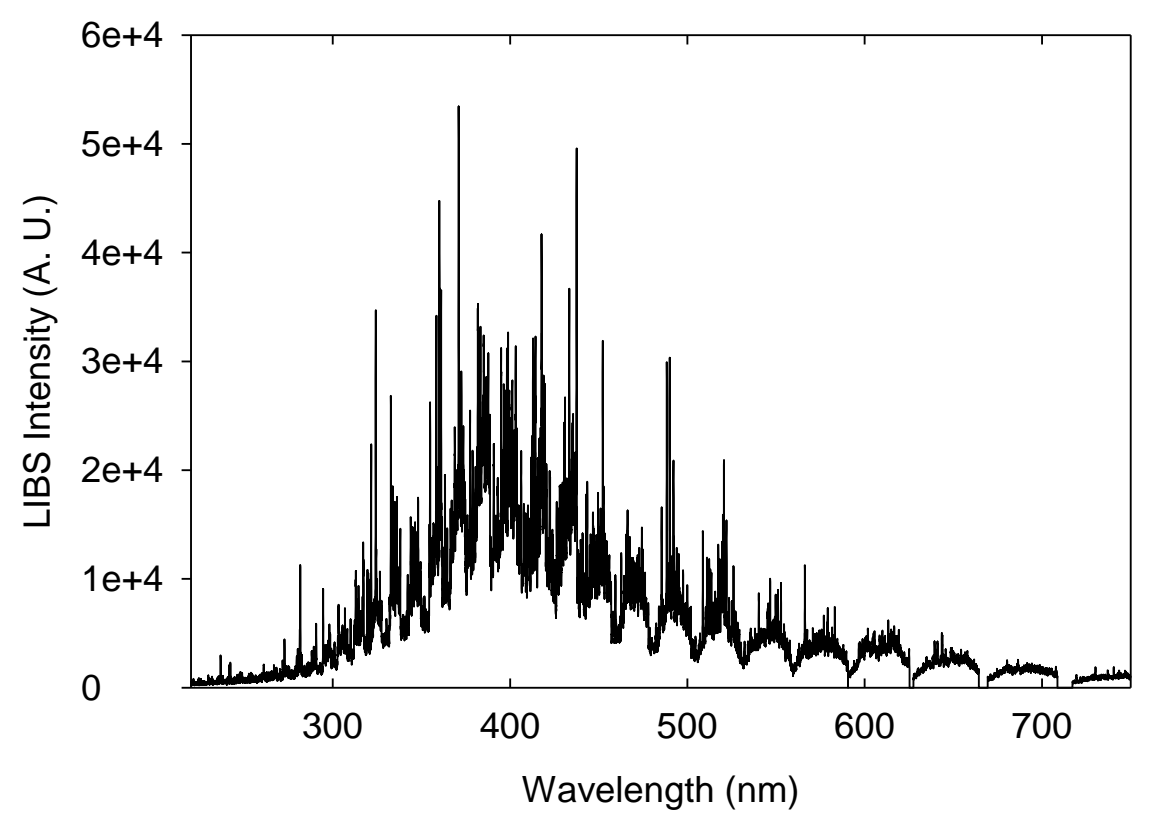

Figure 2(a). LIBS spectrum for a mixture of equal masses of $\mathrm{Eu}, \mathrm{Gd}, \mathrm{La}, \mathrm{Nd}, \mathrm{Pr}, \mathrm{Sm}$, and Y oxides.

Figure 2(a) shows the full broad-band spectrum of an equal mixture of Eu, Gd, La, Nd, $\mathrm{Pr}, \mathrm{Sm}$, and $\mathrm{Y}$ oxides. The concentration of each oxide is $14.3 \%$ (elemental concentrations range from $11.3 \% \mathrm{Y}$ to $12.4 \% \mathrm{Gd}$ ). After acquiring the data for this mixture, it was noticed that the complexity and richness of the spectral features increased greatly, as expected. To see if we can differentiate all seven rare earths within this complex mixture, the spectrum is expanded within the wavelength region of 220-300 $\mathrm{nm}$ in figure 2(b). After expanding the broadband spectrum to focus on a smaller window, it was observed that individual peaks of $\mathrm{Eu}$, $\mathrm{Pr}$, and $\mathrm{Y}$ could be resolved. Further investigation of the wavelength region between 350-400 nm (figure 2(c)) demonstrates thatall seven elements can be identified in this region. Similarly, all seven elementscan be identified in the wavelength region between 350-450 nm (figure 2 (c and d)). 


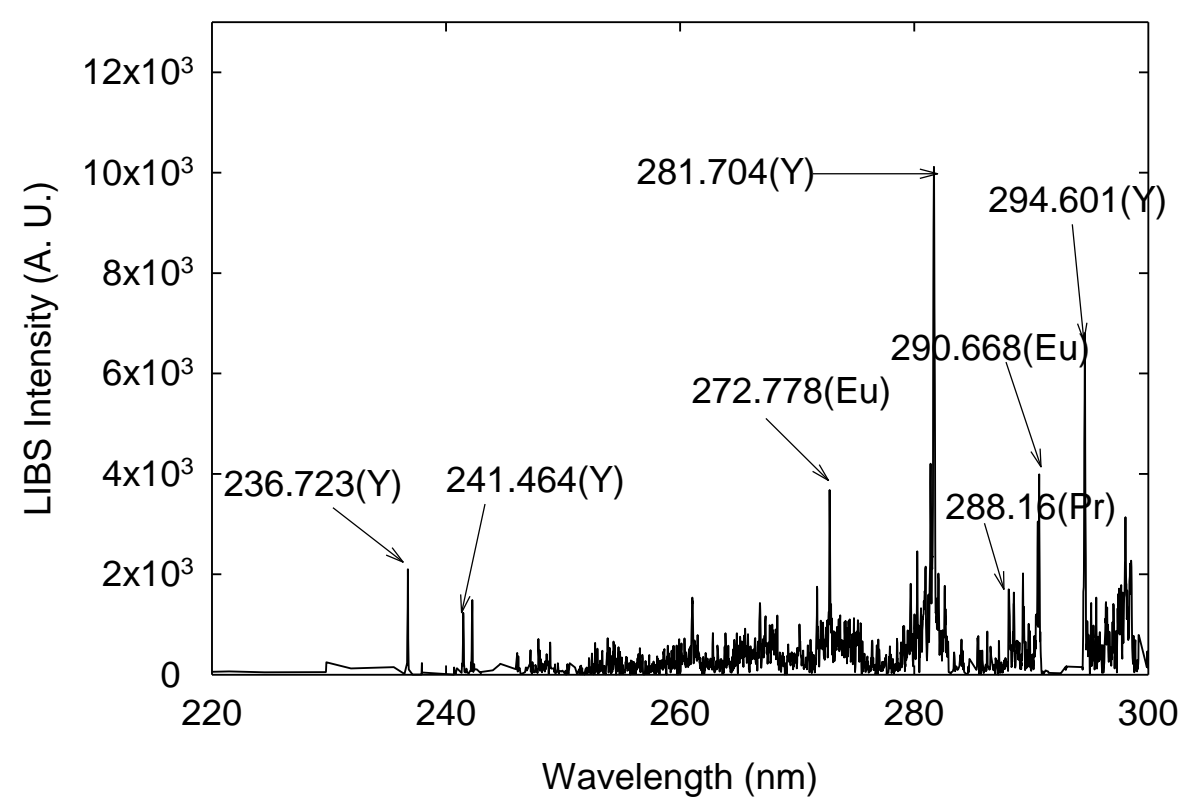

Figure 2(b). LIBS spectrum for amixture of equal masses of $\mathrm{Eu}, \mathrm{Gd}, \mathrm{La}, \mathrm{Nd}, \mathrm{Pr}, \mathrm{Sm}$, and $\mathrm{Y}$ oxides expanded into a wavelength window from $220-300 \mathrm{~nm}$.

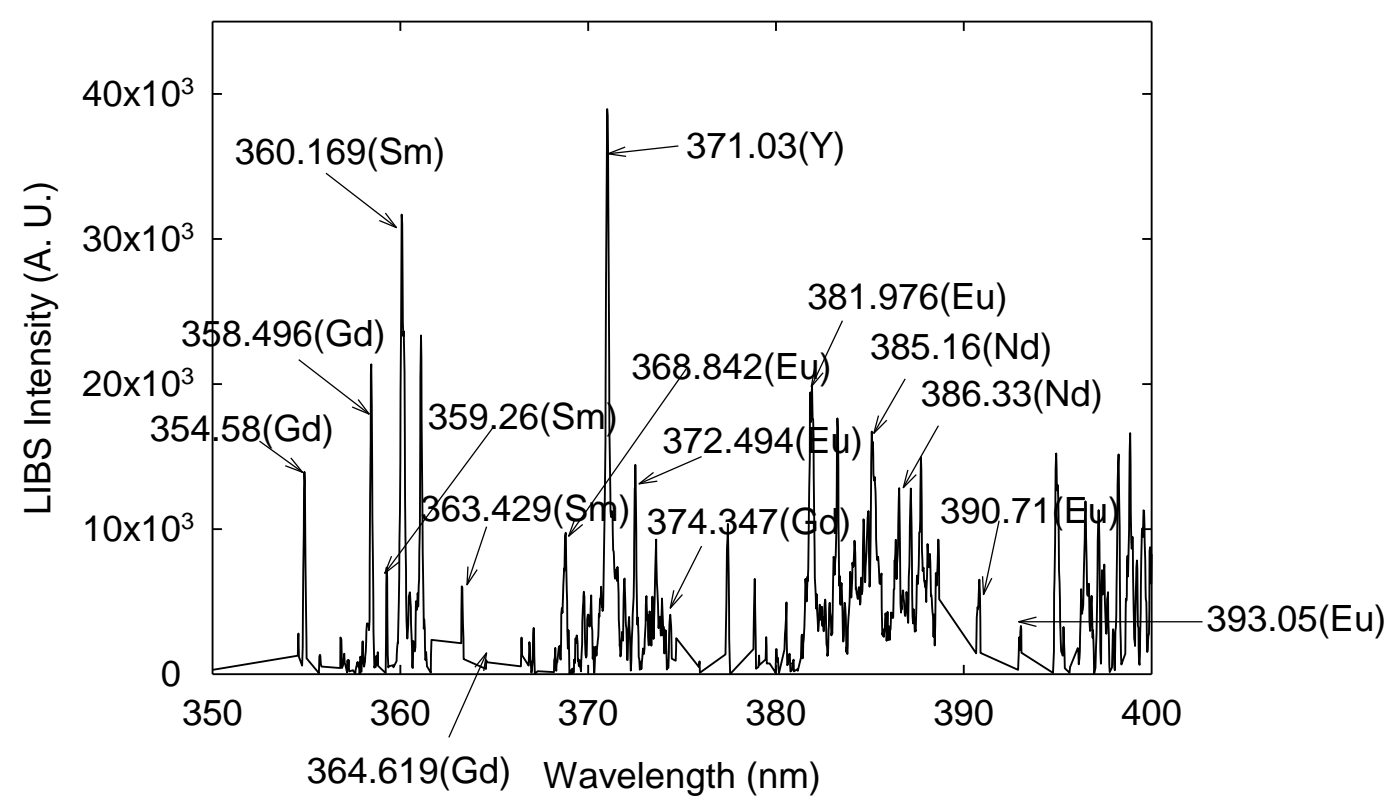


Figure 2(c). LIBS spectrum for amixture of equal masses of Eu, Gd, La, $\mathrm{Nd}, \mathrm{Pr}, \mathrm{Sm}$, and Y oxides expanded into a wavelength window from 350-400 nm

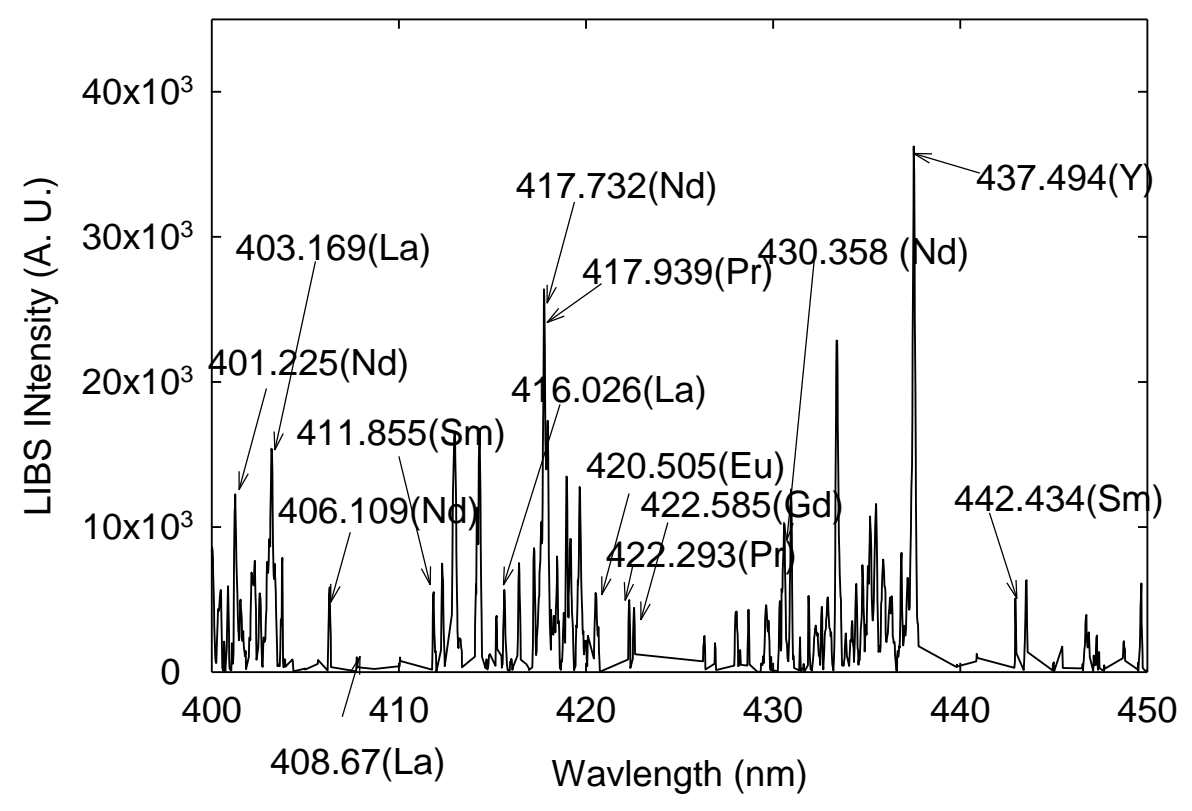

Figure 2(d). LIBS spectrum for a mixture of equal masses of $\mathrm{Eu}, \mathrm{Gd}, \mathrm{La}, \mathrm{Nd}, \mathrm{Pr}, \mathrm{Sm}$, and Y oxides expanded into a wavelength window from 400-450 nm.

Armed with this information, it was concluded that univariate analysis is impractical when analyzing complex mixtures such as those reported in this article. Picking the best spectral features from each element is a tedious and time-consuming task, especially if developing calibration curves for each individual element and then extrapolating that information to theentire mixture of rare earths. In the case of each of the seven elements, six spectra of concentrations each from $1 \%-50 \%$ were obtained for each rare earth oxides mixed with the balance ofgraphite powder. Hence partial least square (PLS) was used for the element concentration determination. PLS is a multivariate analysis (MVA) technique that provides a model for the relationship between a set of predictor variables $\mathrm{X}$ ( $n$ objects, $m$ variables) and a 
set of response variables $\mathrm{Y}$ ( $n$ objects, $p$ responses) [49]. In this case, $m$ variables are the LIBS wavelengths and $p$ responses are properties such as the concentrations for the six rare earths and one transition metal. The $p$ response must be independently measured for each sample. If the spectral data contain information about the properties of interest, a reliable calibration model can be constructed. Multivariate analysis of the data is performed using the Unscrambler (version 9.7) software, CAMO, Corvallis, OR[50]. Three important results in using this approach are (1) the $r^{2}$ coefficient of determination for the calibration and validation, (2) the loading parameters for each element which should be examinedin detail to verify if the features that were selected by the PLS model are real peaks attributed to the specific elements, and (3) the number of principal components (PCs) that the model uses for prediction of those elements. In general, the lower the number of PCs, the better is the development/construction of the model. If the numbers of PCs are greater than 10 to 15 , then there is the danger of integrating on the features in the noise of the spectra and not on the real peaks. A PLS model is used to determine the correlation between measured and predicted rare earth content from the laser-induced breakdown spectra.Figure 3(a) depicts the predicted versus measuredEu, Gd, La, and Nd scatter plots for the calibration (all samples) and full cross-validation sample sets. Excellent coefficients of determination $\left(\mathrm{r}^{2}\right)$ were found for the calibration for each element with 0.97 , and 0.95 , and 0.97, and 0.98, respectively. Full cross-validation results were slightly lower with $0.95,0.91$, 0.93 , and 0.97 respectively. 

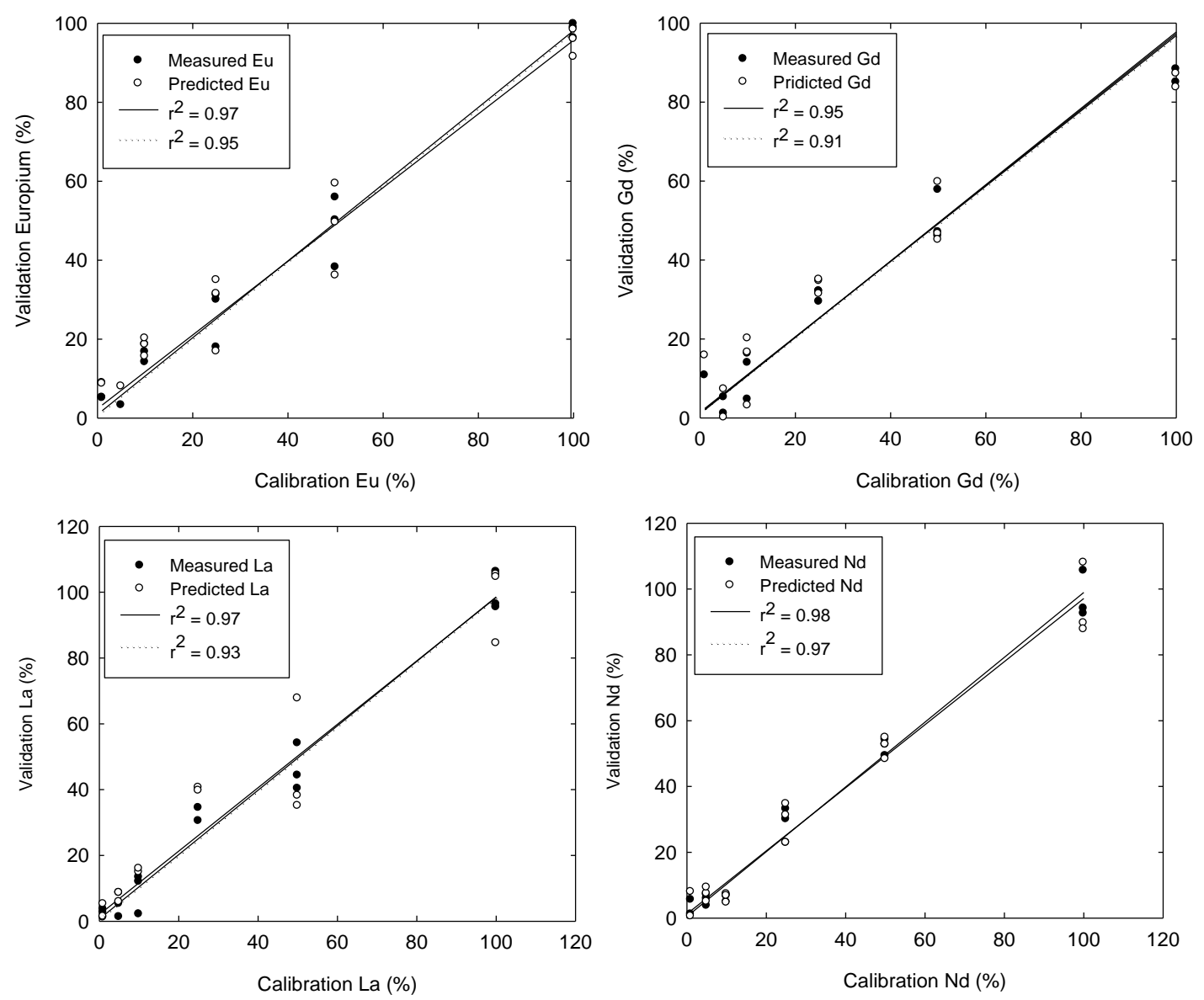

Figure 3(a). $\mathrm{r}^{2}-$ coefficient of determination for calibration and full cross-validation sample sets for $\mathrm{Eu}, \mathrm{Gd}, \mathrm{La}$, and $\mathrm{Nd}$.

Figure 3(b) depicts the predicted versus measured multivariate regression curves for $\operatorname{Pr}$, Sm, and Y scatter plots for the calibration and full cross-validation sample sets. Excellent coefficients of determination $\left(\mathrm{r}^{2}\right)$ were found for the calibration sample set for each element with $0.99,0.97$, and 0.99 , respectively. Full cross-validation results were slightly weaker (especially 
for $\operatorname{Pr}$ and $\mathrm{Sm}$ ), with $0.87,0.89$, and 0.97 , respectively.
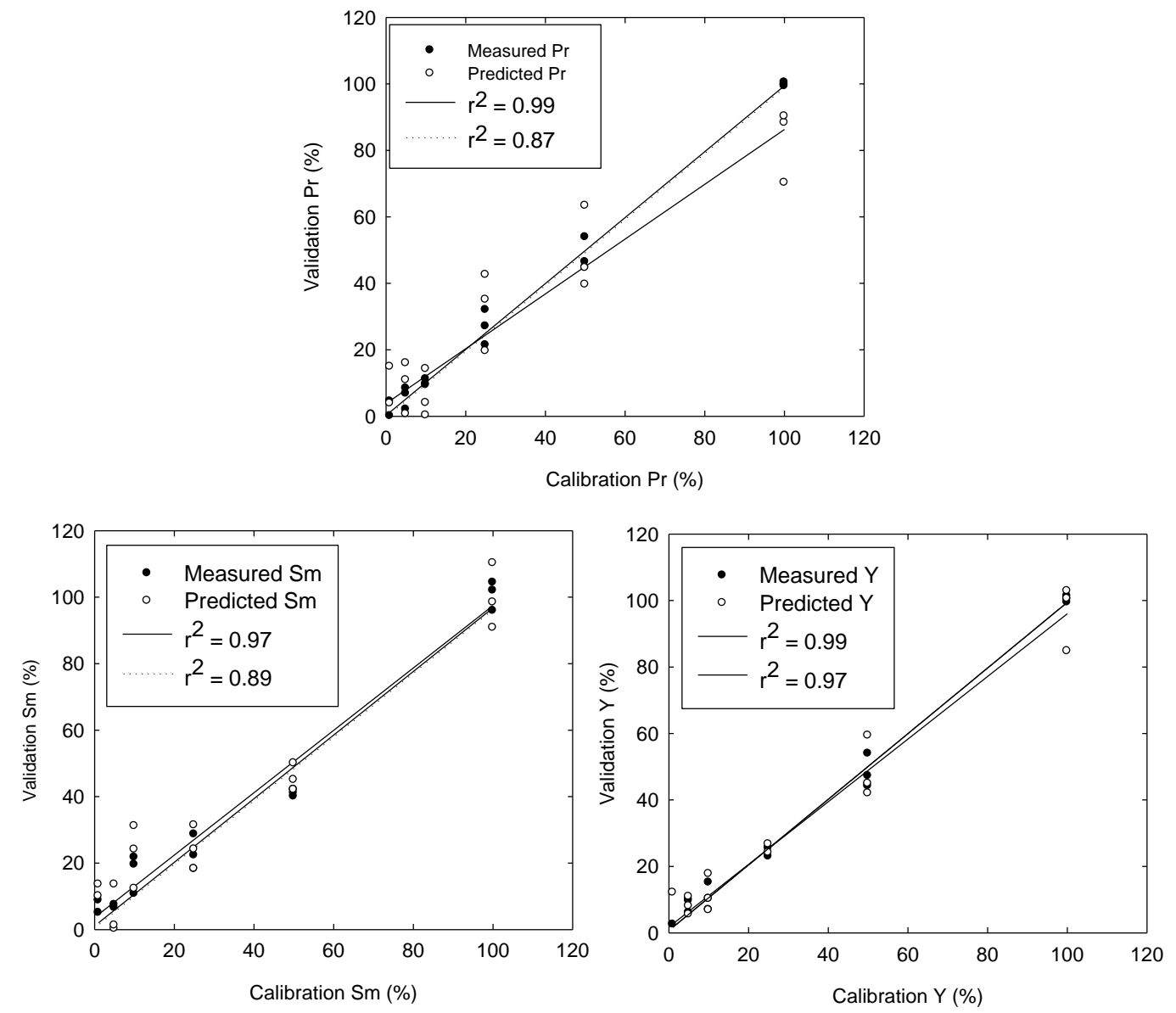

Figure 3(b). $\mathrm{r}^{2}$ - coefficient of determinationfor calibration and full cross-validation for $\mathrm{Pr}, \mathrm{Sm}$, and $\mathrm{Y}$.

PLS regression models help identify the spectral features present in these rare earth oxide mixturesthat contribute to thePLS regression models, in which case acceptable regression coefficients are obtained for both calibration and validation models.No pre-treatment of data was undertaken. Out of the total six spectra that were acquired for each element at each concentration, three were used for calibration and the remaining three were used for validation. PLS1 was performed along with calibration and full cross-validation. PCA was done to find the 
outliers for each element and those were eliminated from the calibration set. This is shown in Table2. below.

Table 2.Regression coefficients, offsets, and root mean square error (RMSE) for both calibration and validation sets for $\mathrm{Eu}, \mathrm{Gd}, \mathrm{La}, \mathrm{Nd}, \mathrm{Pr}, \mathrm{Sm}$, and Y.

\begin{tabular}{|l|l|l|l|l|}
\hline Element & Slope & Offset & RMSE & R-Square \\
\hline Eu (Calibration) & 0.973 & 0.87 & 5.73 & 0.973 \\
\hline Eu (Validation) & 0.933 & 2.37 & 8.09 & 0.951 \\
\hline Gd (Calibration) & 0.954 & 1.47 & 7.43 & 0.954 \\
\hline Gd (Validation) & 0.967 & 1.06 & 10.4 & 0.913 \\
\hline La (Calibration) & 0.988 & 0.68 & 5.17 & 0.979 \\
\hline $\mathrm{La}$ (Validation) & 0.964 & 2.05 & 10.06 & 0.929 \\
\hline $\mathrm{Nd}$ (Calibration) & 0.985 & 0.48 & 4.27 & 0.985 \\
\hline $\mathrm{Nd}$ (Validation) & 0.958 & 1.27 & 6.04 & 0.973 \\
\hline $\operatorname{Pr}$ (Calibration) & 0.992 & 0.26 & 3.14 & 0.992 \\
\hline $\operatorname{Pr}$ (Validation) & 0.824 & 3.88 & 12.16 & 0.875 \\
\hline $\mathrm{Sm}$ (Calibration) & 0.969 & 1.02 & 6.30 & 0.968 \\
\hline Sm (Validation) & 0.920 & 1.97 & 12.25 & 0.896 \\
\hline $\mathrm{Y}$ (Calibration) & 0.993 & 0.22 & 2.88 & 0.993 \\
\hline $\mathrm{Y}$ (Validation) & 0.944 & 1.62 & 6.75 & 0.969 \\
\hline
\end{tabular}

Figure 4 shows the loading parameters for all seven rare earth and transition elements that have been identified as the contributing features in distinguishing the rare earth and transition elements between each other. 


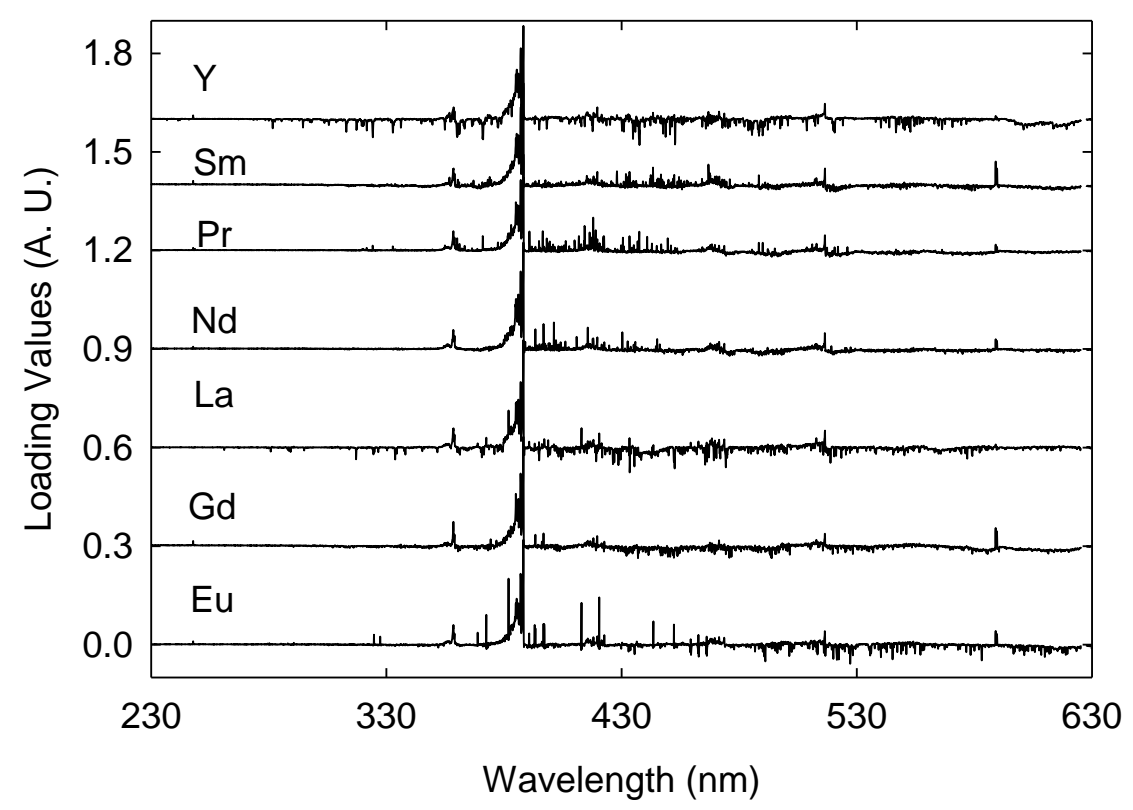

Figure 4.The loading parameters for $\mathrm{Eu}, \mathrm{Gd}, \mathrm{La}, \mathrm{Nd}, \mathrm{Pr}, \mathrm{Sm}$ and $\mathrm{Y}$.

. Table 3 below summarizes the loading parameters for the different elements that the calibration-validation methodology has identified in the wavelength range $390-430 \mathrm{~nm}$.

Table 3.

\begin{tabular}{|l|l|l|l|l|l|}
\hline Element & $\begin{array}{l}\text { Observed } \\
\text { Wavelength } \\
(\mathrm{nm})\end{array}$ & Element & $\begin{array}{l}\text { Observed } \\
\text { Wavelength } \\
(\mathrm{nm})\end{array}$ & Element & $\begin{array}{l}\text { Observed } \\
\text { Wavelength } \\
(\mathrm{nm})\end{array}$ \\
\hline $\mathrm{Eu}(\mathrm{II})$ & 390.71 & $\mathrm{La}(\mathrm{I})$ & 416.026 & $\mathrm{Pr}(\mathrm{II})$ & 422.293 \\
\hline $\mathrm{Eu}(\mathrm{II})$ & 392.887 & $\mathrm{La}(\mathrm{II})$ & 419.655 & $\mathrm{Sm}(\mathrm{II})$ & 392.828 \\
\hline $\mathrm{Eu}(\mathrm{II})$ & 393.048 & $\mathrm{La}(\mathrm{II})$ & 423.838 & $\mathrm{Sm}(\mathrm{II})$ & 411.019 \\
\hline $\mathrm{Eu}(\mathrm{II})$ & 397.196 & $\mathrm{Nd}(\mathrm{II})$ & 392.710 & $\mathrm{Sm}(\mathrm{II})$ & 411.855 \\
\hline $\mathrm{Eu}(\mathrm{II})$ & 397.963 & $\mathrm{Nd}(\mathrm{II})$ & 395.745 & $\mathrm{Sm}(\mathrm{II})$ & 415.221 \\
\hline $\mathrm{Eu}(\mathrm{II})$ & 412.97 & $\mathrm{Nd}(\mathrm{II})$ & 397.330 & $\mathrm{Sm}(\mathrm{II})$ & 419.945 \\
\hline $\mathrm{Eu}(\mathrm{I})$ & 413.707 & $\mathrm{Nd}(\mathrm{II})$ & 397.949 & $\mathrm{Sm}(\mathrm{II})$ & 428.079 \\
\hline $\mathrm{Eu}(\mathrm{II})$ & 420.505 & $\mathrm{Nd}(\mathrm{II})$ & 399.010 & $\mathrm{Y}(\mathrm{II})$ & 393.066 \\
\hline $\mathrm{Gd}(\mathrm{II})$ & 396.929 & $\mathrm{Nd}(\mathrm{II})$ & 401.225 & $\mathrm{Y}(\mathrm{II})$ & 395.036 \\
\hline $\mathrm{Gd}(\mathrm{II})$ & 404.684 & $\mathrm{Nd}(\mathrm{II})$ & 402.478 & $\mathrm{Y}(\mathrm{II})$ & 398.260 \\
\hline $\mathrm{Gd}(\mathrm{I})$ & 405.364 & $\mathrm{Nd}(\mathrm{II})$ & 404.080 & $\mathrm{Y}(\mathrm{I})$ & 407.735 \\
\hline $\mathrm{Gd}(\mathrm{I})$ & 405.822 & $\mathrm{Nd}(\mathrm{II})$ & 407.762 & $\mathrm{Y}(\mathrm{I})$ & 410.236 \\
\hline
\end{tabular}




\begin{tabular}{|l|l|l|l|l|l|}
\hline GdI) & 407.870 & Nd(II) & 410.946 & Y(I) & 412.830 \\
\hline Gd(I) & 409.372 & $\mathrm{Nd}($ II) & 415.626 & Y(I) & 414.284 \\
\hline Gd(II) & 418.425 & $\mathrm{Nd}(\mathrm{II})$ & 417.732 & $\mathrm{Y}(\mathrm{II})$ & 417.754 \\
\hline $\mathrm{Gd}(\mathrm{I})$ & 419.078 & $\operatorname{Pr}(\mathrm{II})$ & 391.885 & $\mathrm{Y}(\mathrm{II})$ & 419.928 \\
\hline $\mathrm{Gd}(\mathrm{I})$ & 422.585 & $\operatorname{Pr}(\mathrm{II})$ & 396.426 & & \\
\hline $\mathrm{La}(\mathrm{II})$ & 392.922 & $\operatorname{Pr}(\mathrm{III})$ & 398.051 & & \\
\hline $\mathrm{La}(\mathrm{II})$ & 394.91 & $\operatorname{Pr}(\mathrm{II})$ & 398.968 & & \\
\hline $\mathrm{La}(\mathrm{II})$ & 398.852 & $\operatorname{Pr}(\mathrm{II})$ & 399.585 & & \\
\hline $\mathrm{La}(\mathrm{II})$ & 399.575 & $\operatorname{Pr}(\mathrm{II})$ & 405.880 & & \\
\hline $\mathrm{La}(\mathrm{II})$ & 403.169 & $\operatorname{Pr}(\mathrm{II})$ & 410.072 & & \\
\hline $\mathrm{La}(\mathrm{II})$ & 404.291 & $\operatorname{Pr}(\mathrm{II})$ & 411.389 & & \\
\hline $\mathrm{La}(\mathrm{II})$ & 407.735 & $\operatorname{Pr}(\mathrm{II})$ & 414.311 & & \\
\hline $\mathrm{La}(\mathrm{II})$ & 408.672 & $\operatorname{Pr}(\mathrm{II})$ & 416.804 & & \\
\hline $\mathrm{La}($ (II) & 412.323 & $\operatorname{Pr}(\mathrm{II})$ & 417.225 & & \\
\hline $\mathrm{La}(\mathrm{II})$ & 415.197 & $\operatorname{Pr}(\mathrm{II})$ & 417.939 & & \\
\hline
\end{tabular}

The first observation is that the PLS1 model selected very few features in the spectra as compared to the original dense, complex features.The prominent loading parameters for these elements are shown in figure 4 . The spectral features listed in table 3 are the loading parameters picked out by the program to distinguish the seven elements from each other. The next parameter to be calculated is the total residual variance plot for the seven elements. The total residual variance plot should be a decreasing function of the number of components. Figure 5(a) presents the total residual variance plots and is used to assess where the model structure stops and the noise starts for all the correlations. For example, the first graph is the distribution of Eu variation between the different PCs. The second graph in figure 5(a) shows the distribution of the variation of Gddata between the different PCs, and the third graph is the distribution of the variation of Ladata between the different PCs. All the graphs show that most of the variations in the features that contribute to the residual validation model lie within the PC1 components andless variation is found between PC1 and PC2. Even less variation of the features that contribute to the residual validation model is found between PC2 and PC3, and then the variation 
decreases as expected between PC3 and PC4. This continues until no variation is observed for higher order PCs. The basic PCselection assumesthat inclusionof "small" variations do not correspond to significant data structure(s), but rather to noise. That is why it is important to have fewer PCs to develop a robust model.
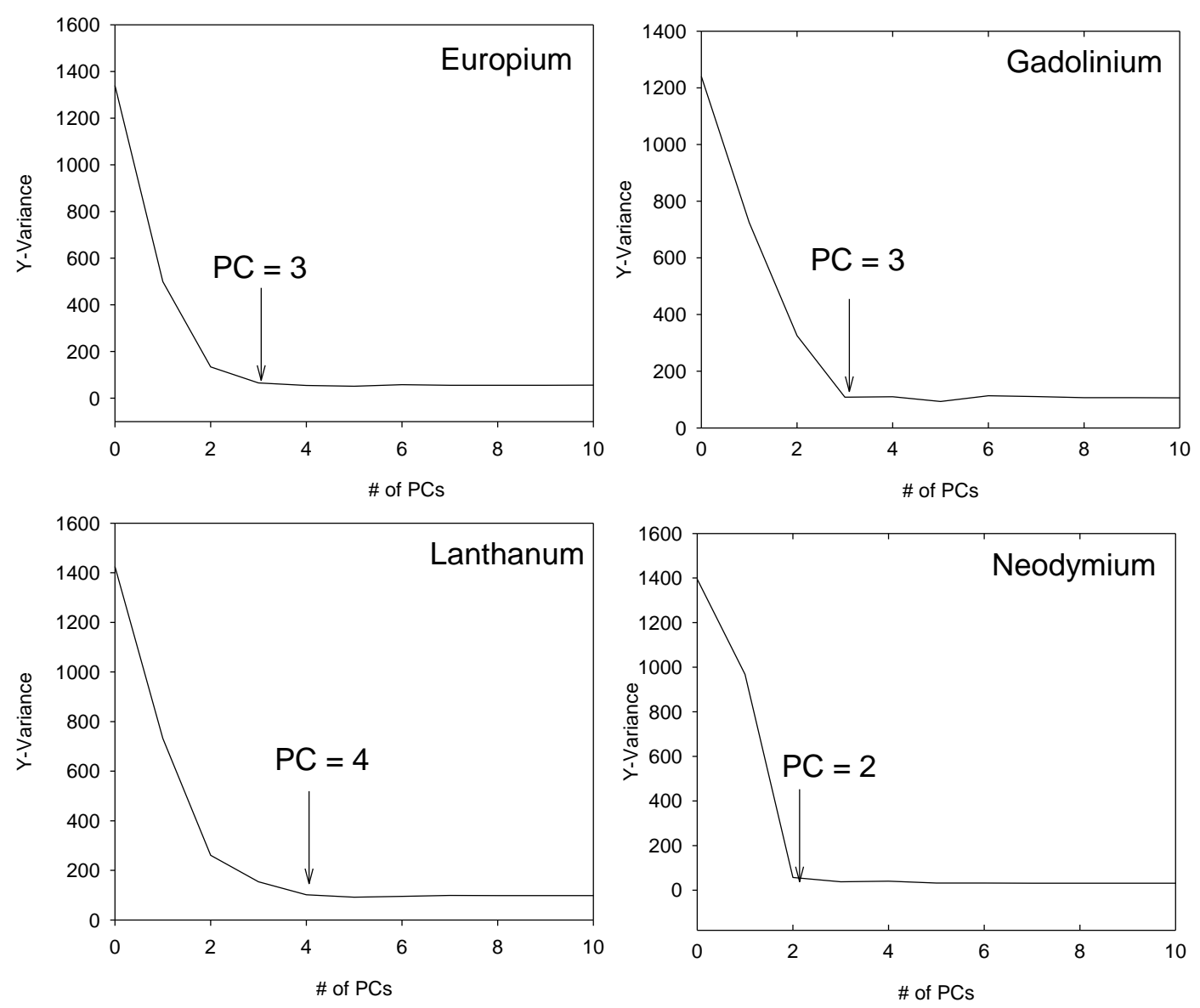

Fig. 5(a).Distribution of Eu, Gd, La, and Ndvariation between the different principal components (PCs).

Similarly in figure 5(b), the first graphdepicts the PC variation reduced to background for PC=6 and in the other graphs the $\mathrm{PC}$ number is 4 for samarium and finally for yttrium the $\mathrm{PC}=4$ at which the variation is completely stabilized. 

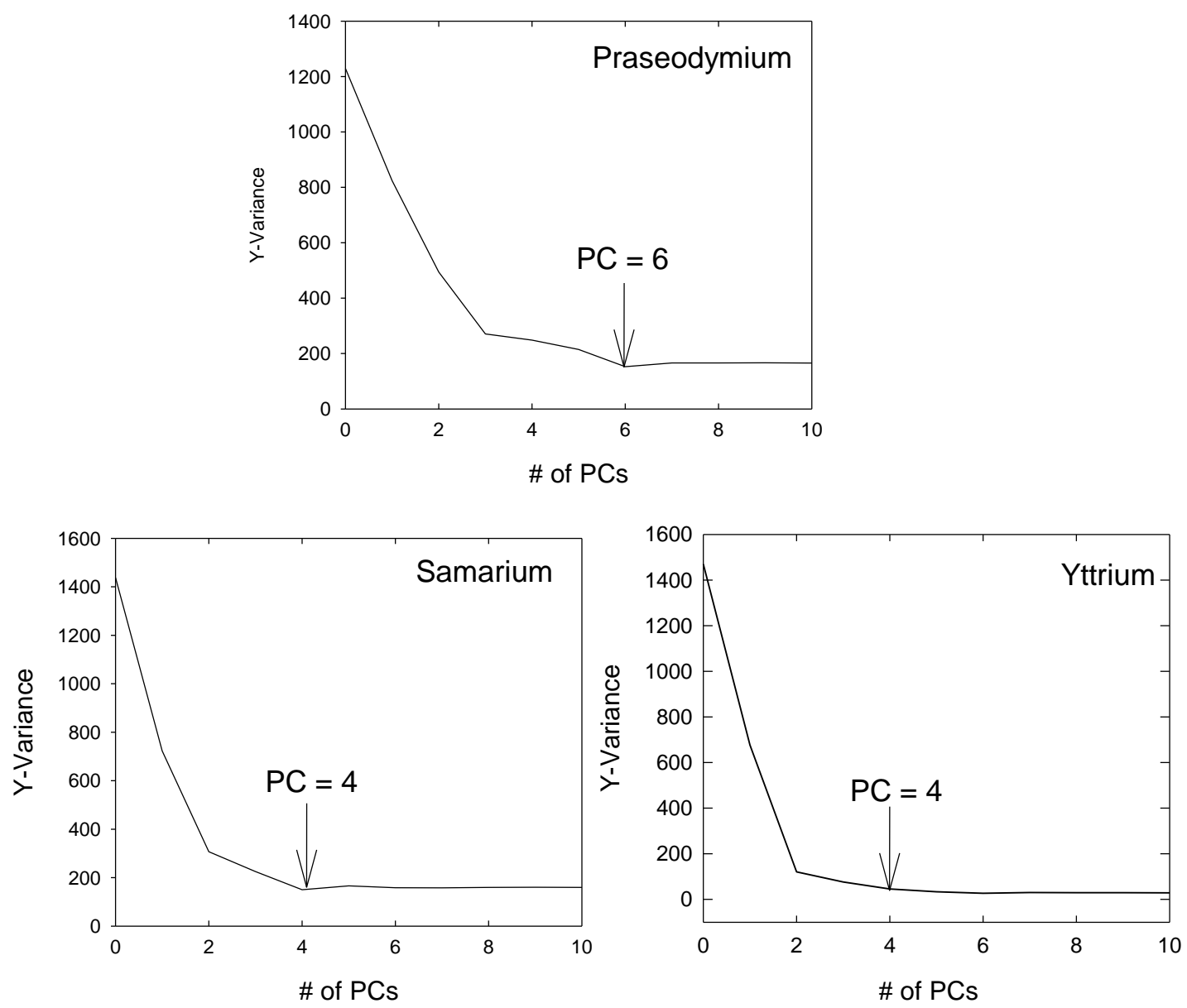

Fig. 5(b).Distribution of Pr, Sm, and Y variation between the different principal components (PCs).

\section{Conclusions}

LIBS was used to obtain the complex emission spectra fromsingle-element samples of six rare earth elements $\mathrm{Eu}, \mathrm{Gd}, \mathrm{La}, \mathrm{Nd}, \mathrm{Pr}$, and $\mathrm{Sm}$, and one transition metal $\mathrm{Y}$, at concentrations of $1 \%$ and $50 \%$ by weight for each element in oxide form. Multivariate calibration and crossvalidation correlations were developed for each individual element. The calibration $\mathrm{r}^{2}$ for each 
element were very good, with values ranging from 0.95 to 0.99 . The cross-validation $r^{2}$ for each element were slightly lower, with values ranging from 0.87 to 0.97 which is to be expected. A multivariate approach was developed for each element of interest and was able to determine those features that were the primary contributors to the mixed spectra from each specific element. The variation of all the elements between different principal components was found to be between 3 and 6 components, hence confirming that the statistical methodology is very robust and stable. Finally, the loading parameters were confirmed as picked by the PLS multivariate analysis model.

\section{Acknowledgements}

This research was supported by the U.S. Department of Energy. This manuscript has been authored by UT-Battelle LLC under Contract No. DE-AC05-00OR22725 with the U.S. Department of Energy. The United States Government retains and the publisher, by accepting the article for publication, acknowledges that the United States Government retains a non-exclusive, paid-up, irrevocable, world-wide license to publish or reproduce the published form of this manuscript, or allow others to do so, for United StatesGovernment purposes. 


\section{References}

[1] S. D. Herrmann, S. X. Li, M. F. Simpson, and S. Phongikaroon, Electrolytic Reduction of Spent Nuclear Oxide Fuel as Part of an Integral Process to Separate and Recover Actinides from Fission Products, Separation Sci. and Technol., 41, (2006) 1965-1983.

[2] Jong-Il Yun, R. Klenze, and Jae-Il Kim, Laser-Induced Breakdown Spectroscopy for the On-line MultielementAnalysis of Highly Radioactive Glass melt. Part I: Characterization and Evaluation of the Method, Appl. Spectrosc., 56(4) (2002) 437-448.

[3] Jong-Il Yun, R. Klenze, and Jae-Il Kim, Laser-Induced Breakdown Spectroscopy for the On-line MultielementAnalysis of Highly Radioactive Glass melt. Part II: Analyses of Molten Glass Samples, Appl. Spectrosc., 56(7) (2002) 852-858.

[4] C. A. Smith, M. A. Martinez, D. K. Veirs, and D. A. Cremers, Pu-239/Pu-240 isotope ratios determined using high resolution emission spectroscopy in a laser-induced plasma”, SpectrochimicaActa B57 (2002) 929-937.

[5] D. A. Cremers, A. Beddingfield, R. Smithwick, R. Chinni, C. R. Jones, B. Beardsley, and L. Karch,Monitoring Uranium, Hydrogen, and Lithium and Their Isotopes Using a Compact Laser-Induced Breakdown Spectroscopy (LIBS)Probe High-Resolution Spectrometer, Appl. Spectrosc., 66(3) (2012) 250-261.

[6] J. E. Barefield II, E. J. Judge, J. M. Berg, S. P. Willson, L. A. Le, and L. N. Lopez, Analysis and Spectral Assignments of Mixed Actinide Oxide Samples Using laserInduced Breakdown Spectroscopy (LIBS), Appl. Spectrosc., 67(4) (2013) 433-440. 
[7] E. J. Judge, J. E. Barefield II, J. M. Berg, S. M. Clegg, G. J. Havrilla, V. M. Montoya, L. A. Le, and L. N. Lopez, 'Laser-induced breakdown spectroscopy measurements of uranium and thorium powders and uranium ore," SpectrochimicaActa B,83-84 (2013) 2836.

[8] T. Bundschuh, Jong-Il Yun, and R. Knopp, "Determination of size, concentration and elemental composition of colloids with laser-induced breakdown detection/spectroscopy (LIBD/S),” Fresnius J Anal. Chem., 371 (2001) 1063-1069.

[9] D. Alamelu, A. Sarkar, S. K. Aggarwal, Laser-induced breakdown spectroscopy for simultaneous determination of $\mathrm{Sm}, \mathrm{Eu}$, and $\mathrm{Gd}$ in aqueous solutions, Talanta 77 (2008) 256-261.

[10] Jong-Il Yun, T. Bundschuh, V. Neck, and Jae-Il Kim, Selective Determination of Europium (III) Oxide and HydroxideColloids in Aqueous Solution by Laser-Induced Breakdown Spectroscopy, Appl. Spectrosc., 55(3) (2001) 273-278.

[11] E. C. Jung, D. H. Lee, J.-I Yun, J. G. Kim, J. W. Yeon, and K. Song, Quantitative determination of uranium and europium in glass matrix by laser-induced breakdown spectroscopy, SpectrochimicaActa B 66(9-10)(2011) 761-764.

[12] H. Hotokezaka, S. Tanaka, A. Suzuki, and S. Nagasaki, Speciation analysis on europium(III) using laser-induced breakdown spectroscopy, RadiochimicaActa 88(9-10) (2000) 645-648.

[13] D. Menut, P. Mauchien, Y.S. Gif , M. Descostes, P. Meier, J. Radwan, and C. Poinssot, Eu migration in Callovo-Oxfodian argillite: on the use of micro laser-induced breakdown 
spectroscopy (micro LIBS) as a microanalysis tool, Int. conf. on chemistry and migration behavior of actinides and fission products in the geosphere; Avignon (France) vol. 38(27), 1-(Sept. 2005).

[14] G. Zikratov, R. Vasudev, F. Y. Yueh, J. P. Singh, and J. C. Marra, Laser induced breakdown spectroscopy of hafnium doped vitrified glass, Glass Technol., 40(3) (1999) 84-88.

[15] K. Song, D. Kim, H. K. Cha, Y. Kim, E. C. Jung, I. Choi, H. S. Yoo, and S. Oh, Characterization of laser-induced plasma in a vacuum using laser ablation mass spectrometry, and laser-induced breakdown spectrometry, Microchem. J., 76 (1-2) (2004) 95-103.

[16] M. Oba, Y. Maruyama, K. Akaoka, M. Miyabe, I. Wakaida, Double-pulse LIBS of gadolinium oxide ablated by femto- and nano-second laser pulses, Appl. Phys. A 101 (2010) 545-549.

[17] V. K. Unnikrishnan, R. Nayak, P. Devangad, M. M. Tamboli, C. Santhosh, G. A. Kumar, and D. K. Sardar, Calibration based laser-induced breakdown spectroscopy (LIBS) for quantitative analysis of doped rare earth elements in phosphors, Mat. lett, 107 (2013) 322-324.

[18] S. H. Kim, J. S. Ju, Y. H. Lee, J. H. Jeong, and H. S. Sin, Semi-quantitative analysis of the Neodymium binary alloys using Laser induced breakdown spectroscopy, Trans. Korean Nucl. Soc., Autumn meeting PueongChang, Korea Oct 30-31 (2008). 
[19] S. J. Rehse and C. A. Ryder, Laser-induced breakdown spectroscopy for branching ratio and atomic lifetime measurements in singly-ionized neodymium and gallium, SpectrochimicaActa B., 64(10) (2009) 974-980.

[20] A. F. M. Y. Haider, M. A. Rony, R. S. Lubna, and K. M. Abedin, Detection of multiple elements in coal samples from Bangladesh by laser-induced breakdown spectroscopy, Optics \& Laser Technol., 43(8) (2011) 1405-1410.

[21] S. H. Kim, J. S. Hong, J. S. Ju, and H. S. Sin, A quantitative analysis for Lanthanum series element using Laser induced breakdown spectroscopy, Trans. Korean Nucl. Soc., Spring meeting, Gyeongju, Korea May29-30 (2008).

[22] A. Ciucci, V. Palleschi, S. Rastelli, R. Barbini, F. Colao, R. Fontini, A. Palucci, S. Ribezzo, and H. J. L. van der Steen, Trace pollutants anlaysis in soil by a time-resolved laser-induced breakdown spectroscopy technique, Appl. Phys. B, 63(2) (1996) 185-190.

[23] S. H. Kim, J. S. Ju, J. -H. Jeong, H. -S. Sin, and H. -D. Kim,Semi- quantitative analysis for Samarium alloys sample using Laser induced breakdown spectroscopy, Trans. Korean Nucl. Soc., Spring meeting,Jeju, Korea May 22 (2009).

[24] J. Pigosova, A. Kilianova, P. Vojtek, M. Kopcok, and A. Cigan, Preparation of bismuthdoped yttrium iron garnets and their characterization. Proc. of SPIE 6609, M1-M7 2007.

[25] S. G. Buckley, H. A. Johnson, K. R. Hencken, D. W. Hahn. Implementation of laserinduced breakdown spectroscopy as a continuous emissions monitor for toxic metals: Waste Management, 20(5-6) (2002) 455-462. 
[26] H. Zhang, F. -Y. Yueh, and J. P. Singh, laser-induced breakdown spectrometry as a multimetal continuous-emission monitor, Appl. Opt., 38(9) (1999)1459-1466.

[27] K. M. Abedin, A. F. M. Y. Haider, M. A. Rony, and Z. H. Khan, Identification of multiple rare earths and associated elements in raw monazite sands by laser-induced breakdown spectroscopy, Optics \& Laser Technol., 43(1) (2011) 45-49.

[28] Madhavi Z. Martin, Steve Allman, Deanne J. Brice, Rodger C. Martin, Nicolas O. Andre, Exploring laser-induced breakdown spectroscopy for nuclear materials analysis and insitu applications, SpectrochimicaActa Part B. vol 74-75 (2012) 177-183.

[29] M. Benedict, T. H. Pigford, and H. W. Levi, Nuclear Chemical Engineering, $2^{\text {nd }}$ ed.,

[30] P.W.J.M. Boumans, Basic concepts and characteristics of ICP-AES, in: P.W.J.M. Boumans (Ed.), Inductively Coupled Plasma Emission Spectroscopy, Part 1, Methodology, Instrumentation, and Performance, Wiley, New York, 1987, p. 100, Chap. 4.

[31] P.W.J.M. Boumans, J.A. Tielrooy, F.J.M.J. Maessen, Mutual spectral interferences of rare earth elements in inductively coupled plasma atomic emission spectrometry-I. Rational line selection and correction procedure, Spectrochim.Acta Part B 43 (1988) 173 199.

[32] P.W.J.M. Boumans, J.J.A.M. Vrakking, A.H.M. Heijms, Mutual spectral interferences of rare earth elements in inductively coupled plasma atomic emission spectrometry-II Approach to the compilation and use of pseudo physically resolved spectral data, Spectrochim. Acta Part B 43 (1988) 1365-1488. 
[33] P.W.J.M.Boumans,HeZhi Zhuang, J.J.A.M.Vrakking, J.A.Tielrooy, F.J.M.J. Maessen, Mutual spectral interferences of rare earth elements in inductively coupled plasma atomic emission spectrometry-III. Pseudo physically resolved spectral data: complete results and evaluation, Spectrochim. Acta Part B 44 (1989) 31-93.

[34]. B. Huang, X. Wang, P. Yang, H. Ying, S. Gu, Z. Zhang, Z. Zhuang, Z. Sun,B. Li, An atlas of High Resolution Spectra of Rare Earth Elements for ICP-AES, Royal Society of Chemistry, 2000.

[35] N. Daskalova, S. Velichkov, N. Krasnobaeva, P. Slavova, Spectral interferences in the determination of traces of scandium, yttrium and rare earth elements in "pure" rare earth matrices by inductively coupled plasma atomic emission spectrometry. Part I. Cerium, neodymium and lanthanum matrices, Spectrochim. Acta Part B 47 (1992) E1595-E1620.

[36] S. Velichkov, N. Daskalova, P. Slavova, Spectral interferences in the determination of traces of scandium, yttrium and rare earth elements in "pure" rare earth matrices by inductively coupled plasma atomic emission spectrometry. Part II. Praseodymium and samarium, Spectrochim.Acta Part B 48 (1993) E1743-E1789.

[37] N. Daskalova, S. Velichkov, P. Slavova, Spectral interferences in the determination of traces of scandium, yttrium and rare earth elements in "pure" rare earth matrices by inductively coupled plasma atomic emission spectrometry. Part III. Europium, Spectrochim.Acta Part B 51 (1996) 733-768.

[38] S. Velichkov, E. Kostadinova, N. Daskalova, Spectral interferences in the determination of traces of scandium, yttrium and rare earth elements in "pure" rare earth matrices by 
inductively coupled plasma atomic emission spectrometry, Part IV. Lutetium and yttrium.Spectrochim.Acta Part B 53 (1998) 1863-1888.

[39] E. Kostadinova, L. Aleksieva, S. Velichkov, N. Daskalova, Spectral interferences in the determination of traces of scandium, yttrium and rare earth elements in "pure" rare earth matrices by inductively coupled plasma atomic emission spectrometry, Part V.

Gadolinium and Erbium, Spectrochim. Acta Part B 55 (2000) 689-729.

[40] L. Aleksieva, N. Daskalova, S. Velichkov, Spectral interferences in the determination of traces of scandium, yttrium and rare earth elements in "pure" rare earth matrices by inductively coupled plasma atomic emission spectrometry. Part VI. Ytterbium, Spectrochim. Acta Part B 57 (2002) 1341-1350.

[41]. I. Kolibarska, S. Velichkov, N. Daskalova, Spectral interferences in the determination of traces of scandium, yttrium and rare earth elements in "pure" rare earth matrices by inductively coupled plasma atomic emission spectrometry. Part VII - Terbium, Dysprosium, Holmium and Thulium, SpectrochimicaActa Part B 63 (2008) 603-606 McGraw-Hill, N.Y., 1981.

[42] B. C. Windom and D. W. Hahn, Laser ablation- laser induced breakdown spectroscopy (LA-LIBS): A means for overcoming matrix effects leading to improved analyte response, Journal of Analytical Atomic Spectrometry 24 (2009) 1665-1675.

[43] D. W. Hahn and N. Omenetto, Laser-induced breakdown spectroscopy (LIBS), Part I: Review of basic diagnostics and plasmaparticle interactions: Still-challenging issues within the analytical plasma community, Appl. Spectrosc. 64, 335A (2010) (review article) 
[44] D. W. Hahn and N. Omenetto, Laser-induced breakdown spectroscopy (LIBS), part II: review of instrumental and methodological approaches to material analysis and applications to different fields," Applied Spectroscopy, vol. 66, no. 4, pp. 347-419, 2012 (review article).

[45] Bassiotis, A. Diamantopoulou, A. Giannoudakos, F. Roubani-Kalantzopoulou, and M. Kompitsas, "Effects of experimental parameters in quantitative analysis of steel alloy by laser-induced breakdown spectroscopy," SpectrochimicaActa Part B, vol. 56, no. 6, pp. (2001) 671-683.

[46] F. Anabitarte, A. Cobo, and J.M. Lopez-Higuera, Laser-Induced Breakdown Spectroscopy: Fundamentals, Applications, and Challenges, International Scholarly Research Network ISRN Spectroscopy Volume 2012, Article ID 285240, 12 pages doi:10.5402/2012/285240 (review article).

[47] R. A. Multari, L. E. Foster, D. A. Cremers, and M. J. Ferris, "Effect of sampling geometry on elemental emissions in laser-induced reakdown spectroscopy," Applied Spectroscopy, vol. 50, no. 12 (1996) pp. 1483-1499.

[48] L. K. Felker, M. Z. Martin, J. Miller, and R. C. Martin, unpublished data, Oak Ridge National Laboratory, 2003.

[49] http://www.camo.com/introducer/see-inside-multivariate-data-analysis.pdf

[50] H. Martens and T. Naes, Multivariate Calibration, first ed. John Wiley and Sons, New York, 1989. 


\section{List of Figure Captions}

Fig. 1(a).LIBS spectra for $1 \%$ concentration of Eu, Gd, La, Nd, Pr, Sm, and Y oxides individually in a graphite matrix.

Fig. 1(b).LIBS spectra for $25 \%$ concentration of Eu, Gd, La, Nd, Pr, Sm, and Y oxides individually in a graphite matrix.

Fig. 1 (c).LIBS spectra for $25 \%$ concentrations of Eu-Gd, La, Nd, Pr, Sm, and Y oxides in the expanded wavelength window of 390-430 nm.

Fig. 2 (a).LIBS spectrum for a mixture of equal masses of Eu, Gd, La, Nd, Pr, Sm, and Y oxides.

Fig. 2 (b).LIBS spectrum for a mixture of equal masses of Eu, Gd, La, Nd, Pr, Sm, and Y oxides expanded into wavelength window from 220-300 nm.

Fig. 2 (c).LIBS spectrum for a mixture of equal masses of Eu, Gd, La, Nd, Pr, Sm, and Y oxides expanded into a wavelength window from 350-400 nm.

Fig. 2 (d).LIBS spectrum for a mixture of equal masses of Eu, Gd, La, Nd, Pr, Sm, and Y oxides expanded into a wavelength window from 400-450 $\mathrm{nm}$.

Fig. 3 (a). $r^{2}$ - coefficient of determination for calibration and full cross-validation sample sets for $\mathrm{Eu}, \mathrm{Gd}, \mathrm{La}$, and $\mathrm{Nd}$.

Fig. 3 (b). $r^{2}$ - coefficient of determination for calibration and full cross-validation sample sets for Pr, Sm, and Y.

Fig. 4.The loading parameters for $\mathrm{Eu}, \mathrm{Gd}, \mathrm{La}, \mathrm{Nd}, \mathrm{Pr}, \mathrm{Sm}$, and Y. 
Fig. 5 (a).Distribution of $\mathrm{Eu}, \mathrm{Gd}, \mathrm{La}$, and $\mathrm{Nd}$ variation between the different principal components (PCs).

Fig. 5 (b).Distribution of $\mathrm{Pr}, \mathrm{Sm}$, and Y variation between the different principal components (PCs).

\section{List of Tables.}

Table 1.Characteristics of laser and detector/spectrometer.

Table 2. Regression coefficients, offsets and root mean square error (RMSE) for both calibration and validation sets for $\mathrm{Eu}, \mathrm{Gd}, \mathrm{La}, \mathrm{Nd}, \mathrm{Pr}, \mathrm{Sm}$, and $\mathrm{Y}$.

Table 3. Loading parameters for the different elements identified by the calibration-validation methodology in the wavelength range 390-430 $\mathrm{nm}$. 
Table1. Characteristics of laser and detector/spectrometer.

\begin{tabular}{|l|l|l|l|l|l|}
\hline \multicolumn{7}{|c|}{ Laser Characteristics } \\
\hline \hline Wavelength & Energy/pulse & Rep- rate & & & \\
\hline $532 \mathrm{~nm}$ & $45 \mathrm{~mJ}$ & $10 \mathrm{~Hz}$ & \multicolumn{3}{l|}{} \\
\hline Detector/Spectrometer Characteristics & \multicolumn{3}{|c|}{ Pixel resolution (FWHM) nm } \\
\hline $\begin{array}{l}\text { Wavelength } \\
\text { range nm }\end{array}$ & $\begin{array}{l}\text { Practical } \\
\text { spectral } \\
\text { bandwidth }\end{array}$ & & $200-400$ & $400-600$ & $600-800$ \\
\hline $190-800$ & $210-790 \mathrm{~nm}$ & & 0.06 & $0.06-0.08$ & $0.09-0.12$ \\
\hline
\end{tabular}

Table 2.Regression coefficients, offsets, and root mean square error (RMSE) for both calibration and validation sets for $\mathrm{Eu}, \mathrm{Gd}, \mathrm{La}, \mathrm{Nd}, \mathrm{Pr}, \mathrm{Sm}$, and $\mathrm{Y}$.

\begin{tabular}{|l|l|l|l|l|}
\hline Element & Slope & Offset & RMSE & R-Square \\
\hline Eu (Calibration) & 0.973 & 0.87 & 5.73 & 0.973 \\
\hline Eu (Validation) & 0.933 & 2.37 & 8.09 & 0.951 \\
\hline Gd (Calibration) & 0.954 & 1.47 & 7.43 & 0.954 \\
\hline Gd (Validation) & 0.967 & 1.06 & 10.4 & 0.913 \\
\hline La (Calibration) & 0.988 & 0.68 & 5.17 & 0.979 \\
\hline $\mathrm{La}$ (Validation) & 0.964 & 2.05 & 10.06 & 0.929 \\
\hline $\mathrm{Nd}$ (Calibration) & 0.985 & 0.48 & 4.27 & 0.985 \\
\hline $\mathrm{Nd}$ (Validation) & 0.958 & 1.27 & 6.04 & 0.973 \\
\hline $\operatorname{Pr}$ (Calibration) & 0.992 & 0.26 & 3.14 & 0.992 \\
\hline $\operatorname{Pr}$ (Validation) & 0.824 & 3.88 & 12.16 & 0.875 \\
\hline $\mathrm{Sm}$ (Calibration) & 0.969 & 1.02 & 6.30 & 0.968 \\
\hline Sm (Validation) & 0.920 & 1.97 & 12.25 & 0.896 \\
\hline $\mathrm{Y}$ (Calibration) & 0.993 & 0.22 & 2.88 & 0.993 \\
\hline $\mathrm{Y}$ (Validation) & 0.944 & 1.62 & 6.75 & 0.969 \\
\hline
\end{tabular}


Table 3 below summarizes the loading parameters for the different elements that the calibrationvalidation methodology has identified in the wavelength range $390-430 \mathrm{~nm}$.

\begin{tabular}{|l|l|l|l|l|l|}
\hline Element & $\begin{array}{l}\text { Observed } \\
\text { Wavelength } \\
(\mathrm{nm})\end{array}$ & Element & $\begin{array}{l}\text { Observed } \\
\text { Wavelength } \\
(\mathrm{nm})\end{array}$ & Element & $\begin{array}{l}\text { Observed } \\
\text { Wavelength } \\
(\mathrm{nm})\end{array}$ \\
\hline $\mathrm{Eu}(\mathrm{II})$ & 390.71 & $\mathrm{La}(\mathrm{I})$ & 416.026 & $\mathrm{Pr}(\mathrm{II})$ & 422.293 \\
\hline $\mathrm{Eu}(\mathrm{II})$ & 392.887 & $\mathrm{La}(\mathrm{II})$ & 419.655 & $\mathrm{Sm}(\mathrm{II})$ & 392.828 \\
\hline $\mathrm{Eu}(\mathrm{II})$ & 393.048 & $\mathrm{La}(\mathrm{II})$ & 423.838 & $\mathrm{Sm}(\mathrm{II})$ & 411.019 \\
\hline $\mathrm{Eu}(\mathrm{II})$ & 397.196 & $\mathrm{Nd}(\mathrm{II})$ & 392.710 & $\mathrm{Sm}(\mathrm{II})$ & 411.855 \\
\hline $\mathrm{Eu}(\mathrm{II})$ & 397.963 & $\mathrm{Nd}(\mathrm{II})$ & 395.745 & $\mathrm{Sm}(\mathrm{II})$ & 415.221 \\
\hline $\mathrm{Eu}(\mathrm{II})$ & 412.97 & $\mathrm{Nd}(\mathrm{II})$ & 397.330 & $\mathrm{Sm}(\mathrm{II})$ & 419.945 \\
\hline $\mathrm{Eu}(\mathrm{I})$ & 413.707 & $\mathrm{Nd}(\mathrm{II})$ & 397.949 & $\mathrm{Sm}(\mathrm{II})$ & 428.079 \\
\hline $\mathrm{Eu}(\mathrm{II})$ & 420.505 & $\mathrm{Nd}(\mathrm{II})$ & 399.010 & $\mathrm{Y}(\mathrm{II})$ & 393.066 \\
\hline $\mathrm{Gd}(\mathrm{II})$ & 396.929 & $\mathrm{Nd}(\mathrm{II})$ & 401.225 & $\mathrm{Y}(\mathrm{II})$ & 395.036 \\
\hline $\mathrm{Gd}(\mathrm{II})$ & 404.684 & $\mathrm{Nd}(\mathrm{II})$ & 402.478 & $\mathrm{Y}(\mathrm{II})$ & 398.260 \\
\hline $\mathrm{Gd}(\mathrm{I})$ & 405.364 & $\mathrm{Nd}(\mathrm{II})$ & 404.080 & $\mathrm{Y}(\mathrm{I})$ & 407.735 \\
\hline $\mathrm{Gd}(\mathrm{I})$ & 405.822 & $\mathrm{Nd}(\mathrm{II})$ & 407.762 & $\mathrm{Y}(\mathrm{I})$ & 410.236 \\
\hline $\mathrm{GdI})$ & 407.870 & $\mathrm{Nd}(\mathrm{II})$ & 410.946 & $\mathrm{Y}(\mathrm{I})$ & 412.830 \\
\hline $\mathrm{Gd}(\mathrm{I})$ & 409.372 & $\mathrm{Nd}(\mathrm{II})$ & 415.626 & $\mathrm{Y}(\mathrm{I})$ & 414.284 \\
\hline $\mathrm{Gd}(\mathrm{II})$ & 418.425 & $\mathrm{Nd}(\mathrm{II})$ & 417.732 & $\mathrm{Y}(\mathrm{II})$ & 417.754 \\
\hline $\mathrm{Gd}(\mathrm{I})$ & 419.078 & $\operatorname{Pr}(\mathrm{II})$ & 391.885 & $\mathrm{Y}(\mathrm{II})$ & 419.928 \\
\hline $\mathrm{Gd}(\mathrm{I})$ & 422.585 & $\operatorname{Pr}(\mathrm{II})$ & 396.426 & & \\
\hline $\mathrm{La}(\mathrm{II})$ & 392.922 & $\operatorname{Pr}(\mathrm{III})$ & 398.051 & & \\
\hline $\mathrm{La}(\mathrm{II})$ & 394.91 & $\operatorname{Pr}(\mathrm{II})$ & 398.968 & & \\
\hline $\mathrm{La}(\mathrm{II})$ & 398.852 & $\operatorname{Pr}(\mathrm{II})$ & 399.585 & & \\
\hline $\mathrm{La}(\mathrm{II})$ & 399.575 & $\operatorname{Pr}(\mathrm{II})$ & 405.880 & & \\
\hline $\mathrm{La}(\mathrm{II})$ & 403.169 & $\operatorname{Pr}(\mathrm{II})$ & 410.072 & & \\
\hline $\mathrm{La}(\mathrm{II})$ & 404.291 & $\operatorname{Pr}(\mathrm{II})$ & 411.389 & & \\
\hline $\mathrm{La}(\mathrm{II})$ & 407.735 & $\operatorname{Pr}(\mathrm{II})$ & 414.311 & & \\
\hline $\mathrm{La}(\mathrm{II})$ & 408.672 & $\operatorname{Pr}(\mathrm{II})$ & 416.804 & & \\
\hline $\mathrm{La}(\mathrm{II})$ & 412.323 & $\operatorname{Pr}(\mathrm{II})$ & 417.225 & & \\
\hline $\mathrm{La}(\mathrm{II})$ & 415.197 & $\operatorname{Pr}(\mathrm{II})$ & 417.939 & & \\
\hline & & & & & \\
\hline
\end{tabular}




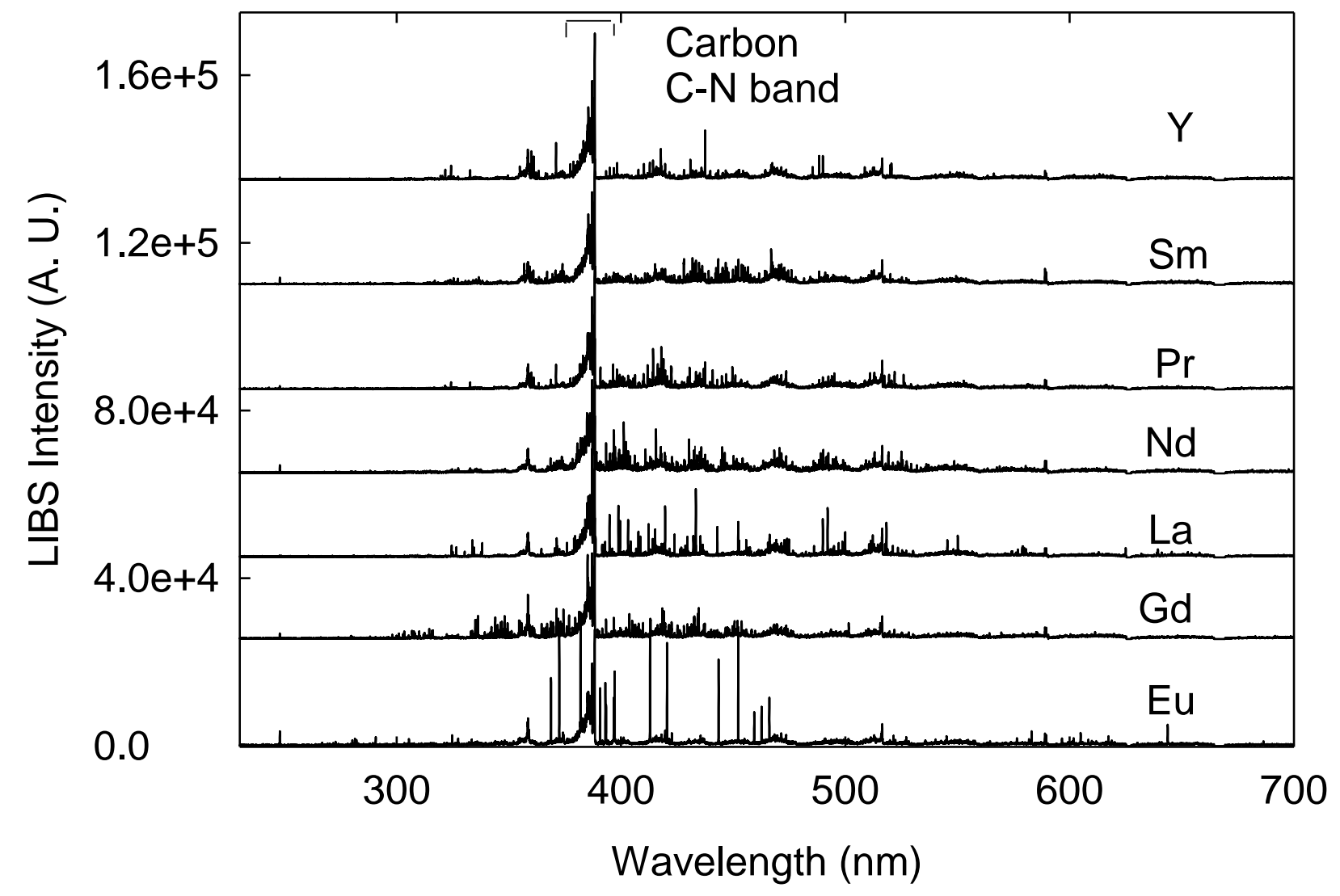

Figure $1(a)$ 


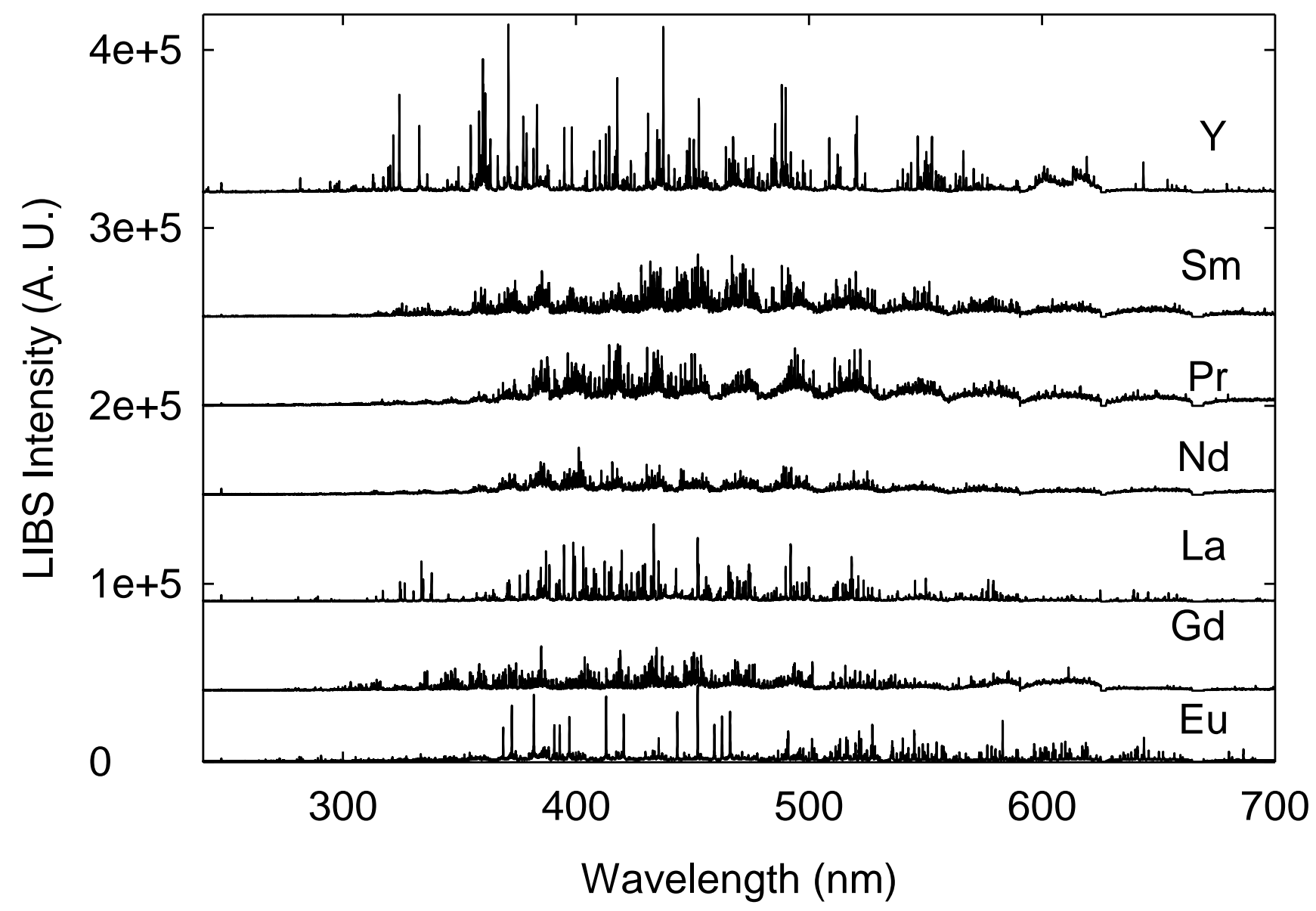

Figure $1(b)$ 


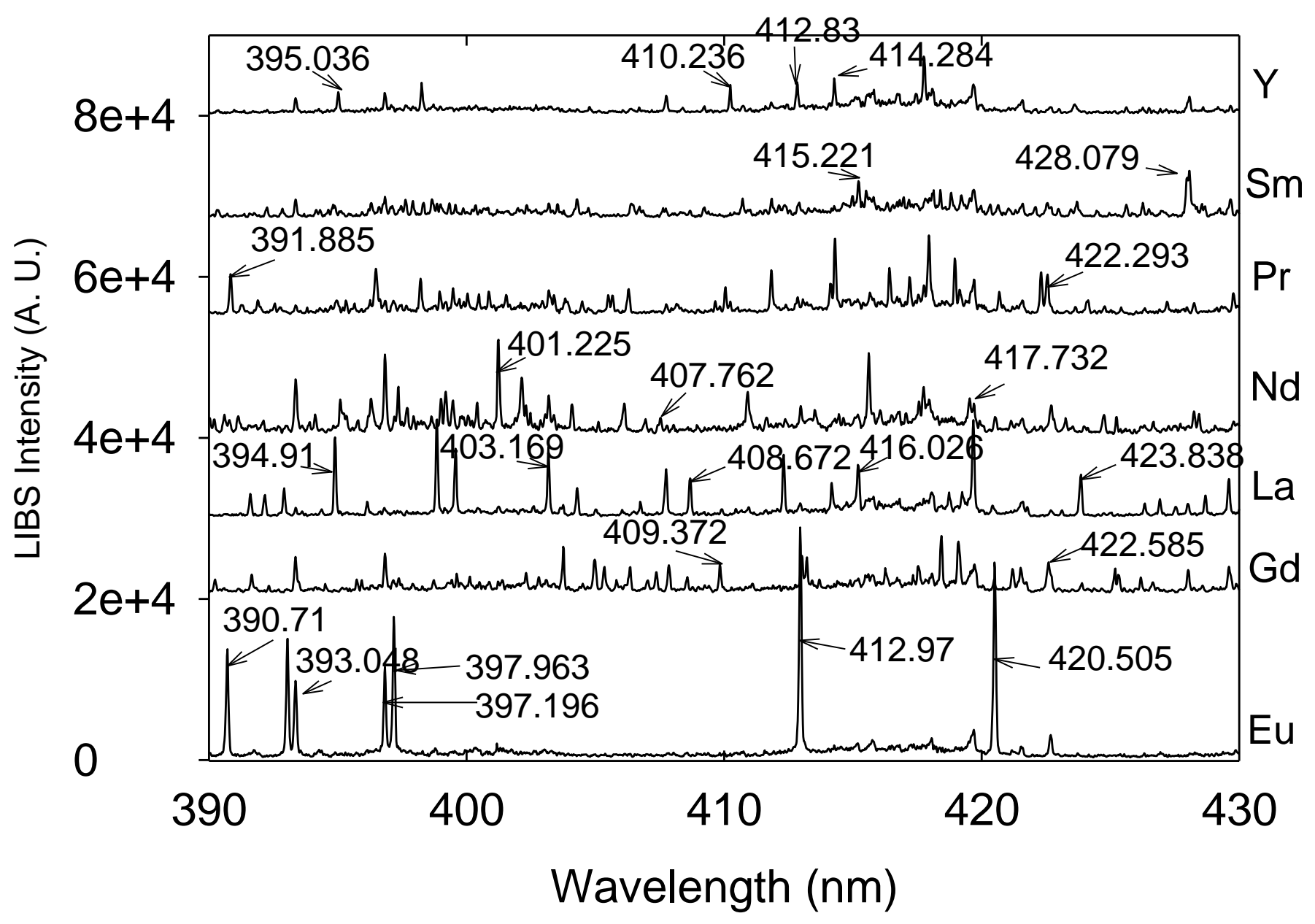

Figure $1(\mathrm{c})$ 


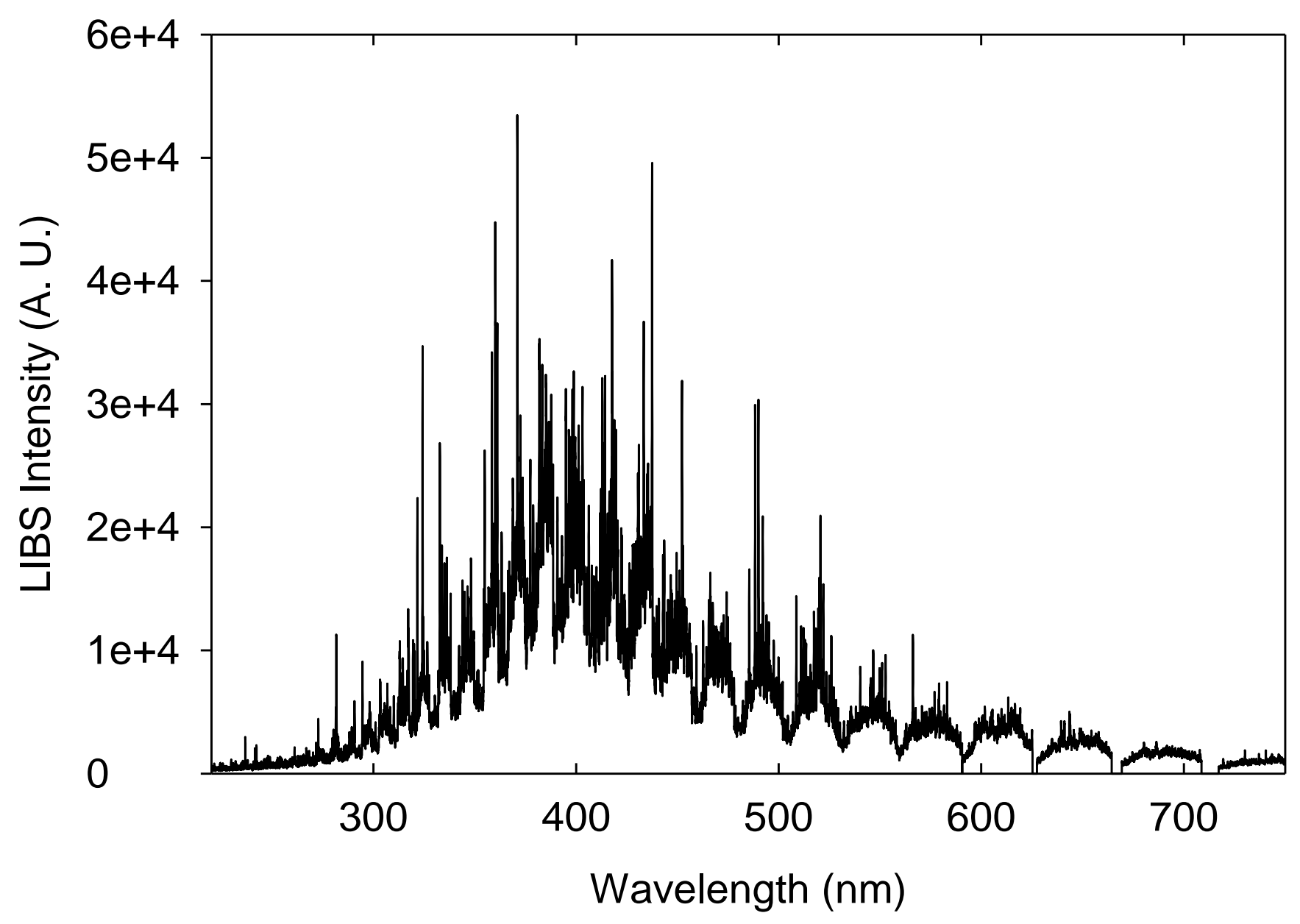

Figure 2(a) 


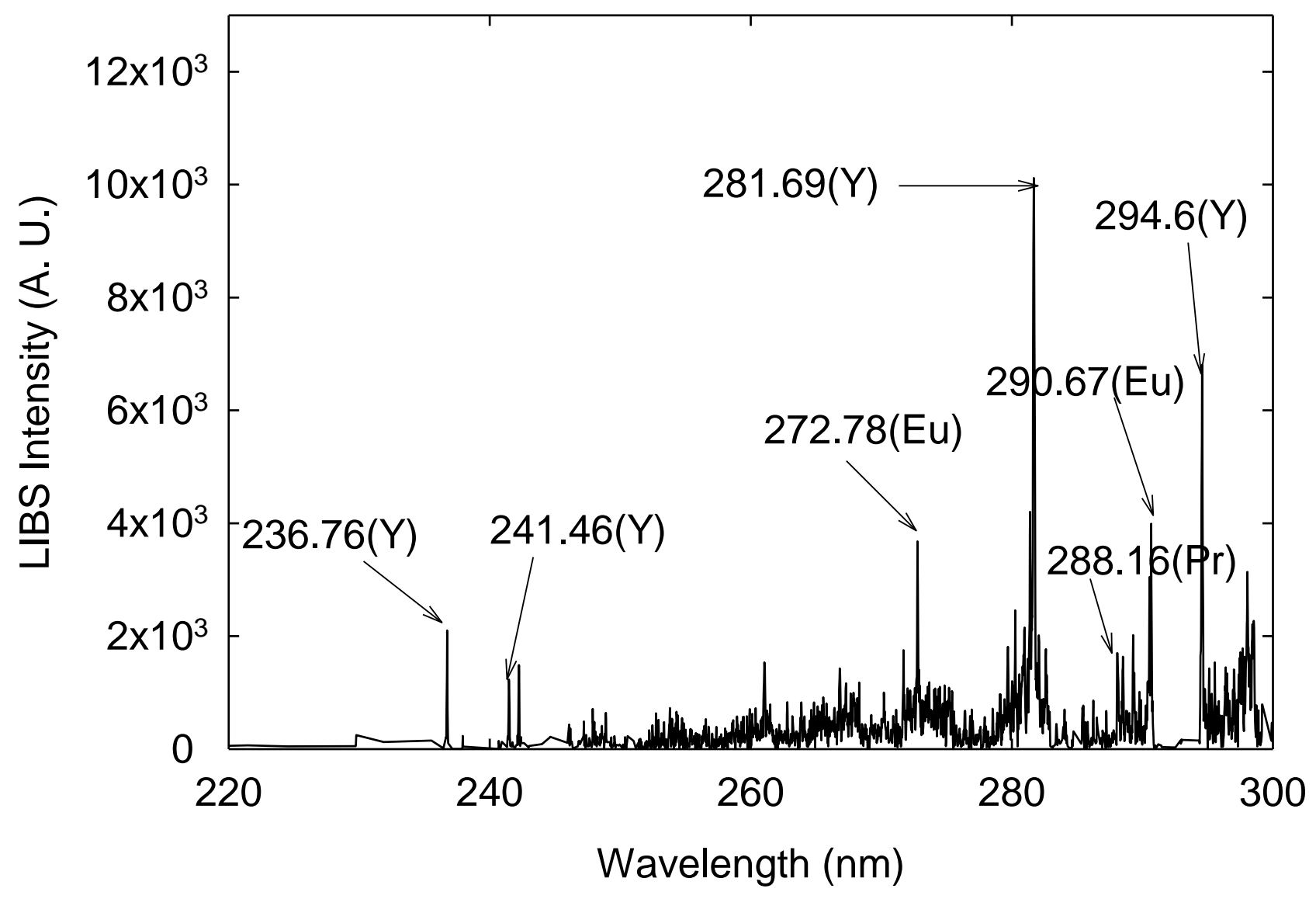

Figure 2(b) 


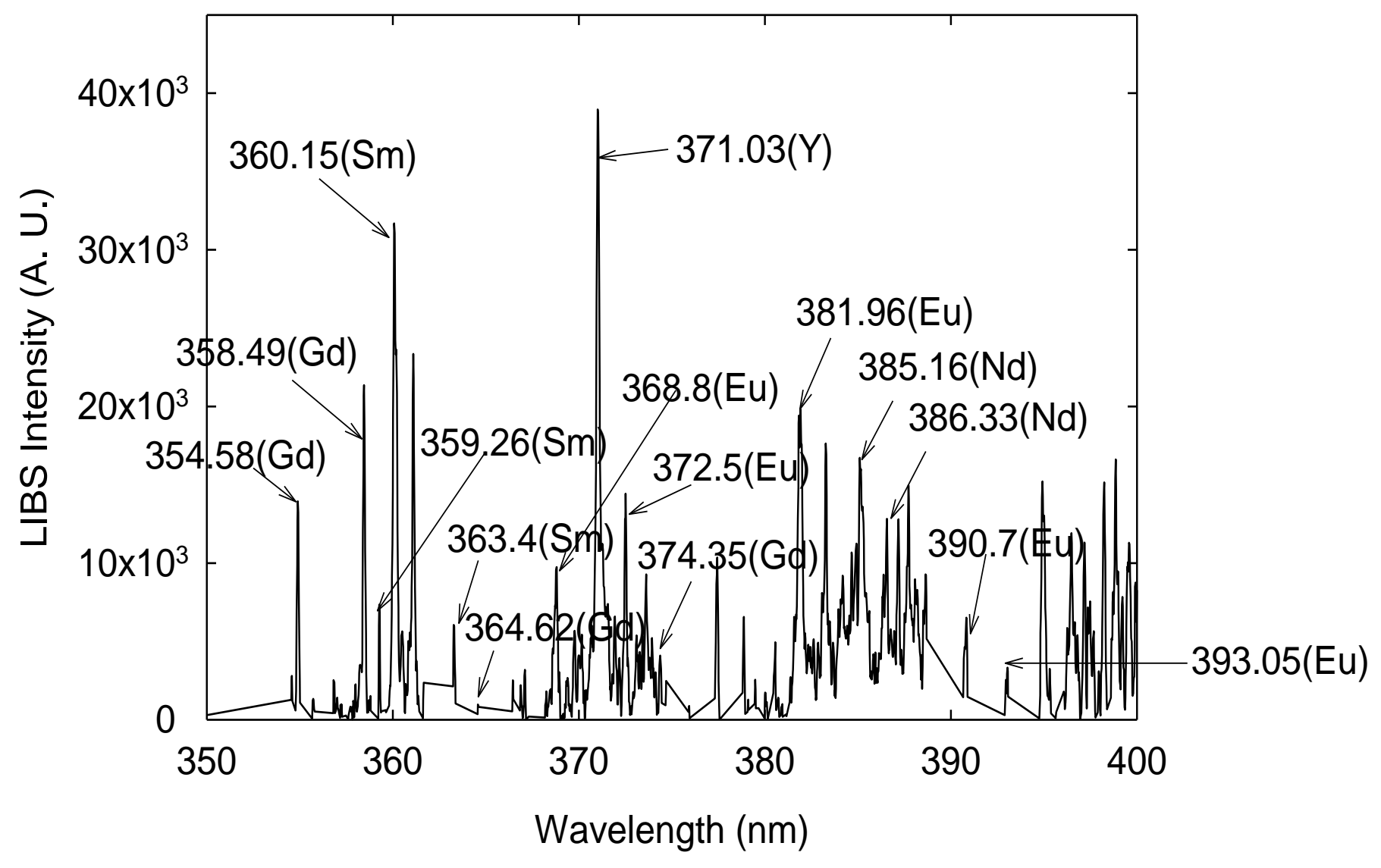

Figure 2(c) 


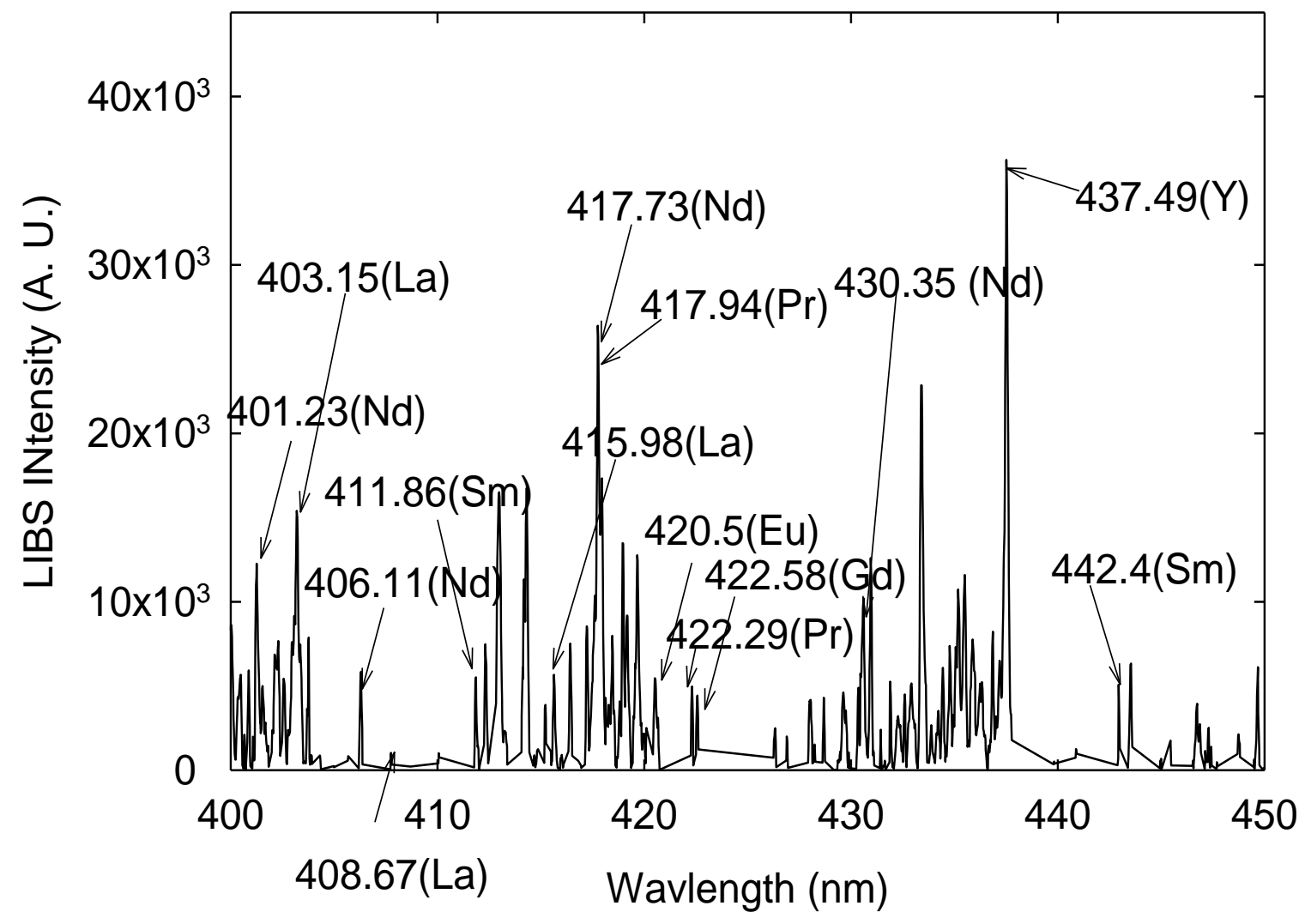

Figure 2(d) 

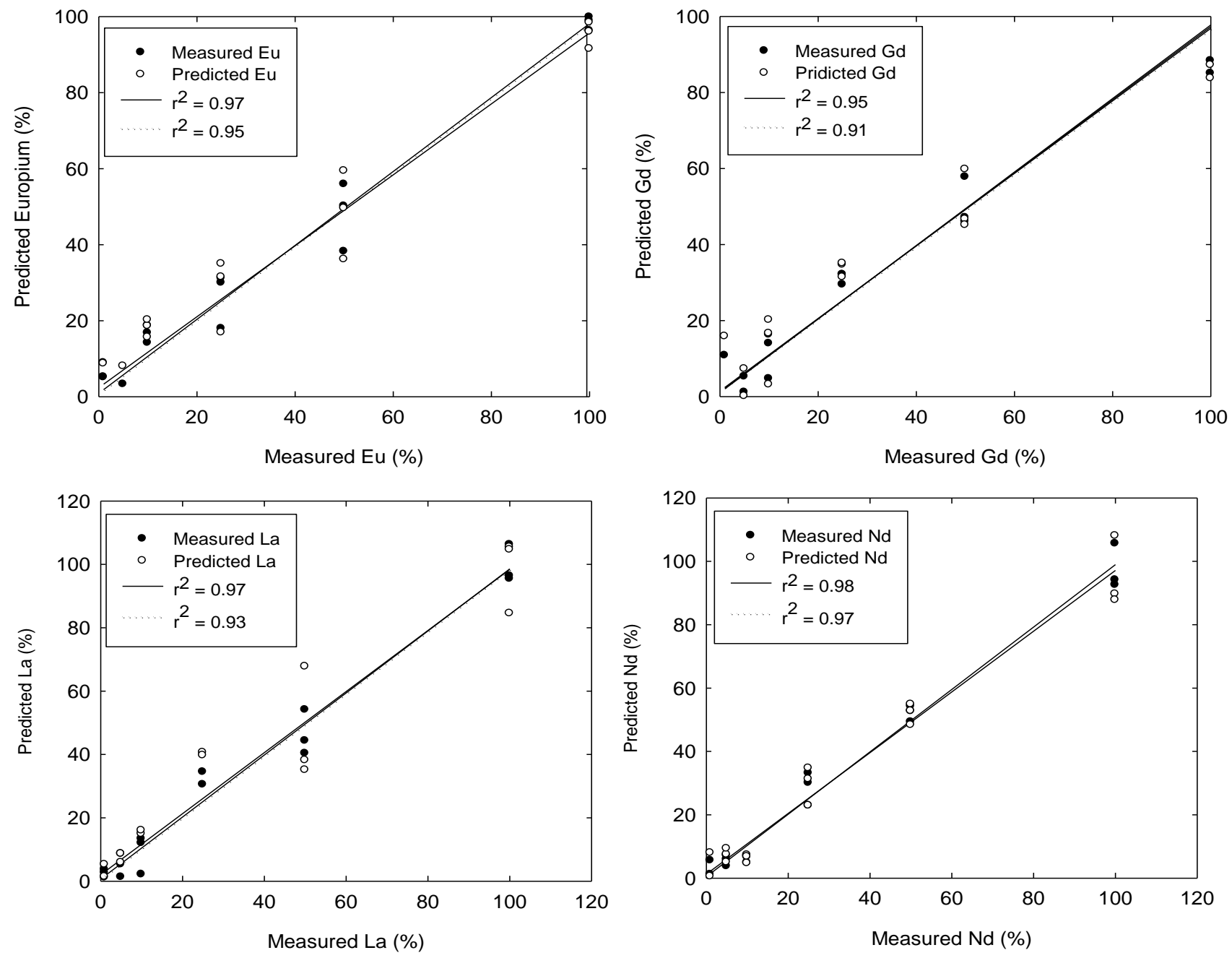

Figure 3(a) 

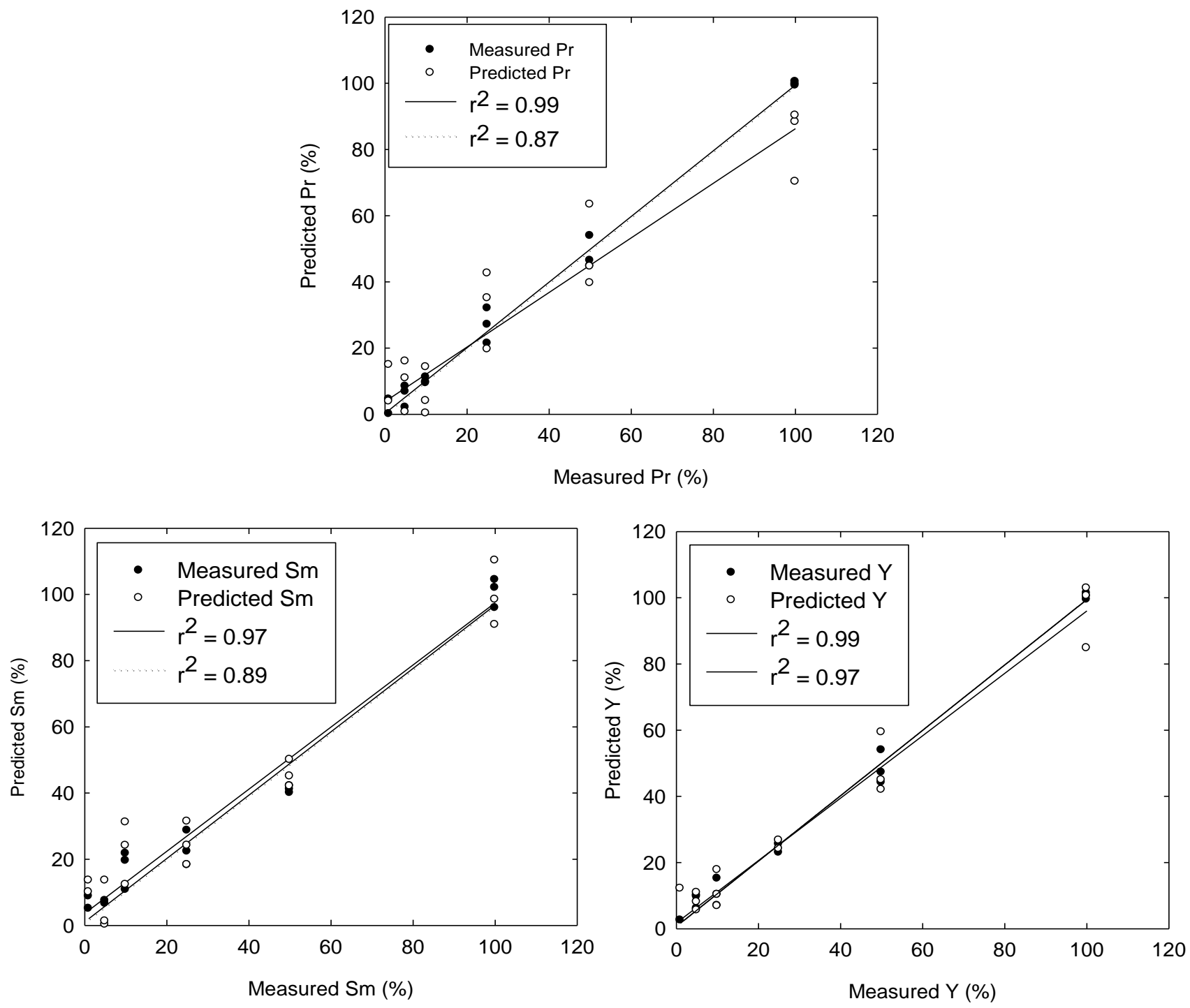

Figure 3(b) 


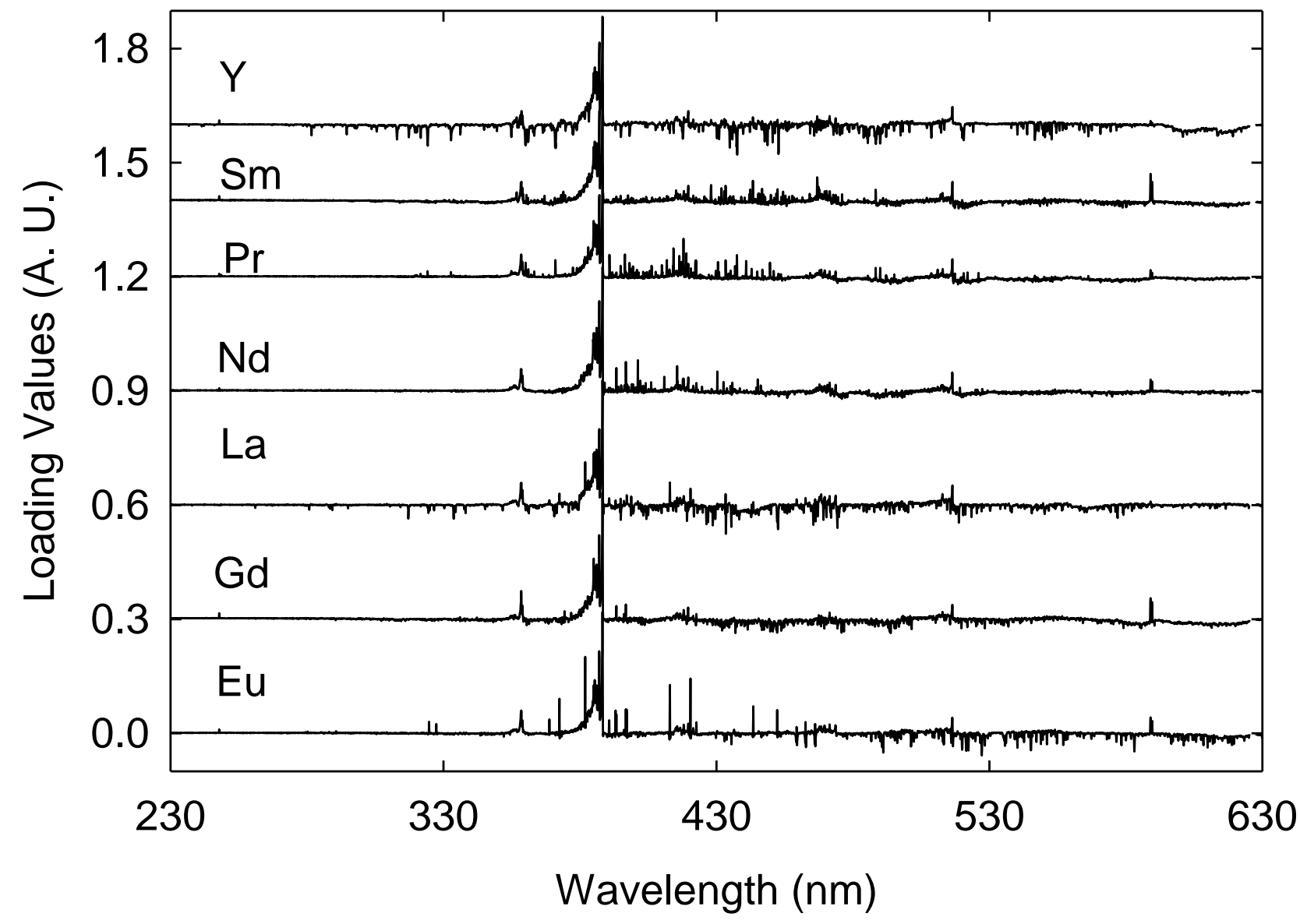

Figure 4 

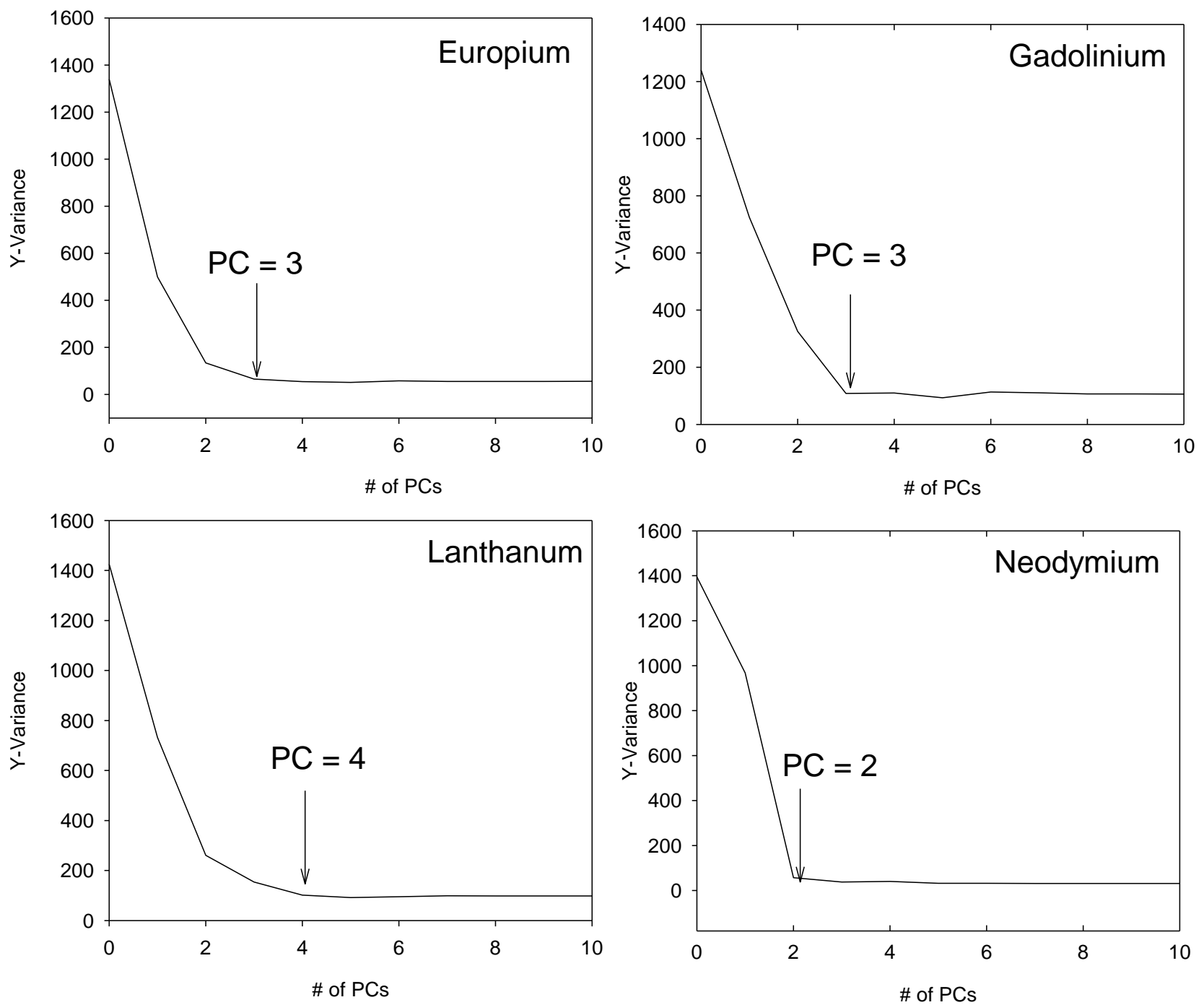

Figure 5(a) 

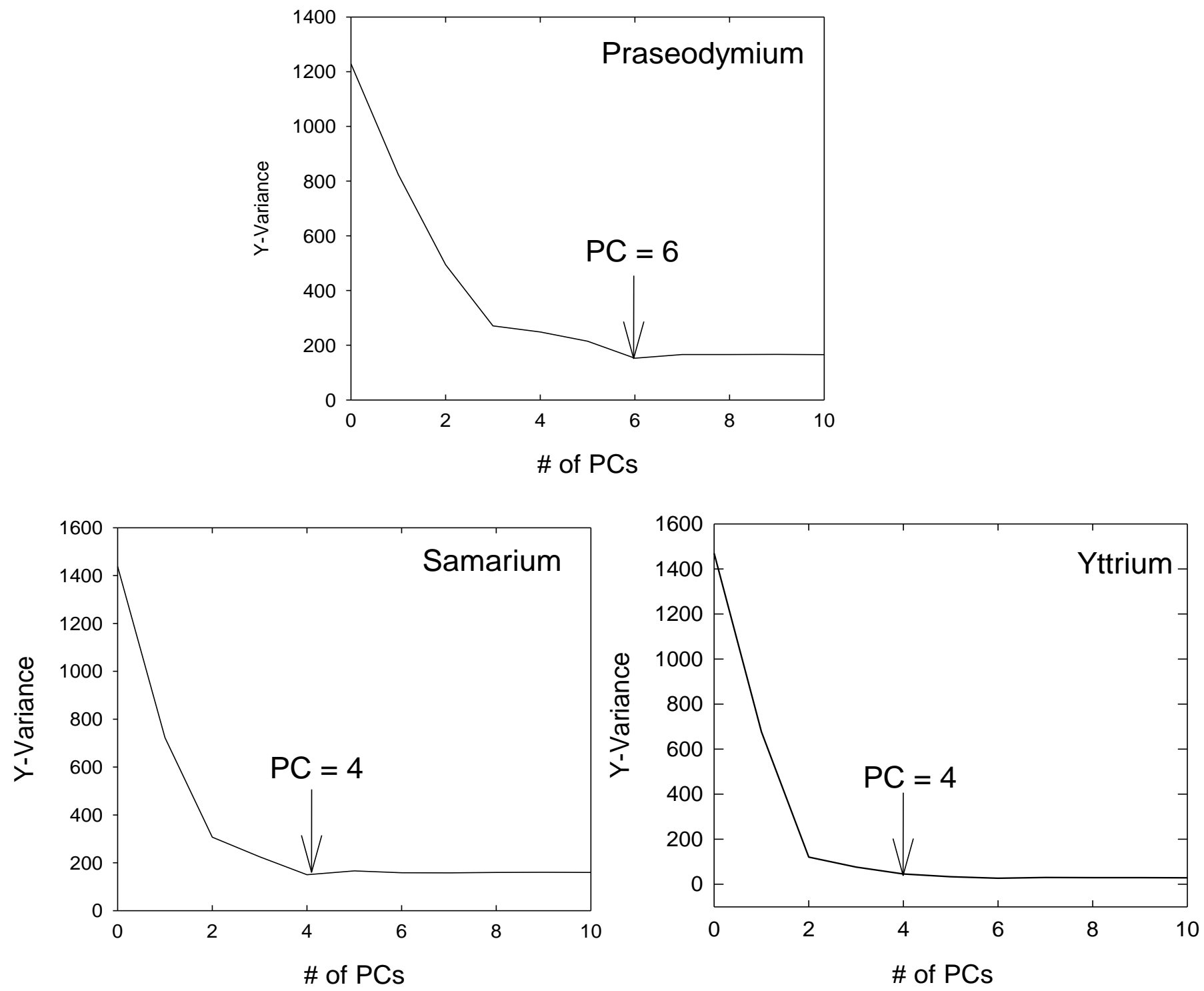

Figure 5(b) 